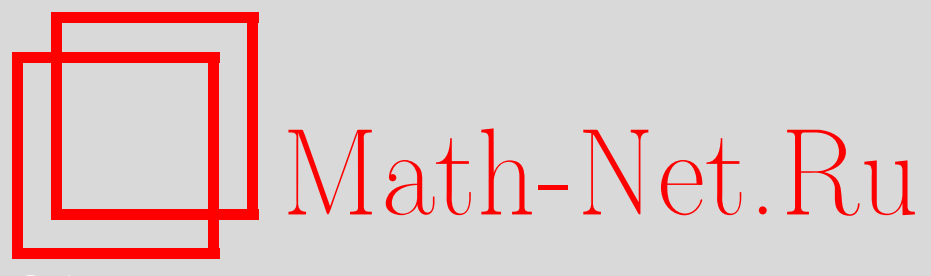

А. Б. Костин, Обратная задача восстановления источника в параболическом уравнении по условию нелокального наблюдения, Матем. сб., 2013, том 204, номер 10, 3-46

DOI: https://doi.org/10.4213/sm8104

Использование Общероссийского математического портала Math-Net.Ru подразумевает, что вы прочитали и согласны с пользовательским соглашением http://www.mathnet.ru/rus/agreement

Параметры загрузки:

IP : 35.174 .16 .151

26 апреля 2023 г., 12:02:37

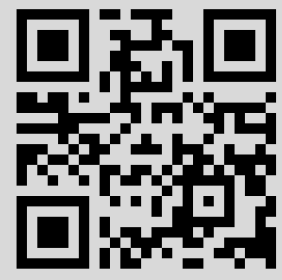




\title{
А. Б. Костин \\ Обратная задача восстановления источника в параболическом уравнении по условию нелокального наблюдения
}

\begin{abstract}
Для параболического уравнения исследуется обратная задача восстановления источника - правой части $F(x, t)=h(x, t) f(x)$, где неизвестной является функция $f(x)$. Для нахождения $f(x)$ помимо начальных и граничных условий задается дополнительное условие нелокального наблюдения вида $\int_{0}^{T} u(x, t) d \mu(t)=\chi(x)$. Для поставленной задачи доказано свойство фредгольмовости, получены достаточные условия существования и единственности ее решения. Эти условия имеют вид легко проверяемых неравенств и не содержат ограничений на величину $T>0$ и диаметр рассматриваемой области $\Omega$. Доказательство основывается на априорных оценках и качественных свойствах решений начально-краевых задач для параболических уравнений.
\end{abstract}

Библиография: 40 названий.

Ключевые слова: обратные задачи, параболические уравнения, нелокальное переопределение.

DOI: $10.4213 / \mathrm{sm} 8104$

\section{§1. Введение}

В работе исследуется обратная задача восстановления правой части в параболическом уравнении. Постановкам и изучению таких задач посвящены работы многих авторов (см., например, [1]-[15]), интерес к ним не ослабевает. Обратные задачи восстановления источника в уравнении можно трактовать как задачи прогноз-управления для систем с распределенными параметрами (см. [16]). Для таких задач, как правило, выделяется класс единственности, в котором ищется решение, и в этом состоит их главное отличие от классических задач оптимального управления. В настоящей работе неизвестный источник имеет вид $F(x, t)=h(x, t) f(x)$, где $f(x)$ - искомая, а $h(x, t)-$ заданная функции (отметим, что такой вид источника в обратных задачах потенциала рассматривался в [1]). Для нахождения функции $f(x)$, помимо начальных и краевых условий (данных прямой задачи), задается дополнительная информация - условие нелокального наблюдения (или переопределения) в виде $l(u) \equiv \int_{0}^{T} u(x, t) d \mu(t)=\chi(x)$ с некоторыми известными мерой $d \mu(t)$ и функцией $\chi(x)$. Для рассматриваемой задачи получены условия однозначной разрешимости, причем в ряде случаев снято условие стационарности эллиптического оператора, входящего в уравнение. 
Задачи с переопределением $l(u)$ финального вида, т.е. $l(u) \equiv u\left(x, t_{1}\right), 0<t_{1} \leqslant$ $T<\infty$, и интегрального вида, т.е.

$$
l(u) \equiv \int_{0}^{T} u(x, t) \omega(t) d t,
$$

для стационарных параболических уравнений в $L_{2}$ рассматривались в [8], [17]. В работах [3], [4] задача с финальным наблюдением рассмотрена в классах Гёльдера, доказано свойство фредгольмовости и теорема единственности, предложен метод, названный потом методом позитивности. Для системы Навье-Стокса свойство фредгольмовости в аналогичной задаче установлено в [18]. Методы исследования задачи с финальным наблюдением развивались в целом ряде работ [3], [5]-[8], [17], в последних двух в единую схему было включено и интегральное наблюдение, в работах [5], [6] применялась теория полугрупп. Более общее условие, а именно

$$
l(u) \equiv \int_{0}^{T} u(x, t) d \mu(t),
$$

возникло чуть позже, см. [10], [11], где линейная обратная задача с таким наблюдением была исследована для уравнений в банаховом пространстве с использованием теории полугрупп и метода позитивности. При этом был обнаружен ряд новых эффектов, например, условие выпуклости вверх функции $\mu(t)$ как достаточное условие единственности (см. [10]). В работе [9] был предложен иной метод доказательства корректности задач с финальным и интегральным наблюдениями, основанный на применении теоремы Крейна-Рутмана. В работах [14] и [15] рассматривалась обратная задача с условием интегрального наблюдения для параболического уравнения в $L_{2}$ с граничными условиями первого рода. Обратные задачи в близкой постановке имеются еще в работе [16], где изучалась связь с сопряженными задачами для абстрактных уравнений, вводились понятия слабого и классического решений и соответствующие понятия корректности обратной задачи. В работах [6], [15], [19] применялся принцип сжимающих отображений, поэтому утверждения этих работ содержат, в отличие от наших результатов, условия малости норм заданных функций. В настоящей статье результаты исследований [8], [9], [17], [20] переносятся на случай более общего переопределения и нестационарного эллиптического оператора в параболическом уравнении. Для доказательства корректности применяется метод работы [9], а единственность обобщенных решений доказывается методом позитивности (см. [3], [8], [12]). Получено описание ядра обратной задачи в случае, когда у задачи отсутствует свойство фредгольмовости. Дополнительные ссылки и комментарии даны в тексте сразу после доказательств.

Рассматриваемые ниже пространства $W_{p}^{2,1}(Q), V_{2}(Q), V_{2}^{1,0}(Q), W_{p}^{2-2 / p}(\Omega)$, $W_{p}^{2}(\Omega), W_{p}^{1}(0, T), L_{q, r}(Q)$ с соответствующими нормами понимаются в общепринятом смысле, все равенства и неравенства понимаются почти всюду (п.в.), а все производные - как обобщенные по С. Л. Соболеву (см., например, [21]), $\mathrm{BV}[0, T]$ - пространство скалярных (вещественных) функций ограниченной вариации на $[0, T]$. Символ $\bigvee_{0}^{T}(\mu)$ будем использовать для полной вариации функции $\mu \in \mathrm{BV}[0, T]$ на отрезке $[0, T]$. Для равенства по определению иногда будем применять обозначение $A:=B$, где слева стоит определяемый объект. 


\section{§ 2. Постановка задачи. Описание результатов}

2.1. Основная задача. Пусть $\Omega \subset \mathbb{R}^{n}$ - ограниченная область с границей $\partial \Omega \in C^{2}$. В цилиндре $Q=\Omega \times(0, T)$ с боковой поверхностью $S=\partial \Omega \times[0, T]$ рассматривается задача о нахождении пары функций $\{\widetilde{u}(x, t) ; \widetilde{f}(x)\}$, удовлетворяющих условиям

$$
\begin{gathered}
\rho(x, t) \widetilde{u}_{t}(x, t)-L(t) \widetilde{u}(x, t)=\widetilde{h}(x, t) \widetilde{f}(x)+\widetilde{g}(x, t), \quad(x, t) \in Q, \\
\widetilde{u}(x, 0)=\widetilde{u}_{0}(x), \quad x \in \Omega, \quad B \widetilde{u}(x, t)=\widetilde{\beta}(x, t), \quad(x, t) \in S, \\
l(\widetilde{u}):=\int_{0}^{T} \widetilde{u}(x, t) d \mu(t)=\widetilde{\chi}(x), \quad x \in \Omega .
\end{gathered}
$$

Здесь функции $\rho, \widetilde{h}, \widetilde{g}, \widetilde{u}_{0}, \widetilde{\beta}, \mu, \widetilde{\chi}$ заданы, равномерно эллиптический оператор $L(t)$ имеет вид

$$
L(t) \widetilde{u}=\sum_{i, j=1}^{n} \frac{\partial}{\partial x_{i}}\left(a_{i j}(x) \frac{\partial \widetilde{u}}{\partial x_{j}}\right)+\sum_{i=1}^{n} b_{i}(x, t) \frac{\partial \widetilde{u}}{\partial x_{i}}+d(x, t) \widetilde{u},
$$

а оператор краевых условий - либо первого, либо третьего рода, т.е.

$$
B \widetilde{u} \equiv \widetilde{u} \quad \text { или } \quad B \widetilde{u} \equiv \frac{\partial \widetilde{u}}{\partial N}+\sigma(x) \widetilde{u}, \quad \text { где } \frac{\partial \widetilde{u}}{\partial N} \equiv \sum_{i, j=1}^{n} \cos \left(\mathbf{n}, x_{i}\right) a_{i j}(x) \frac{\partial \widetilde{u}}{\partial x_{j}}
$$

- производная по конормали, $\mathbf{n}$ - внешняя нормаль к $\partial \Omega$ в точке $x=\left(x_{1}, \ldots, x_{n}\right), \sigma \in C^{1}(\partial \Omega), \sigma(x) \geqslant 0$ на $\partial \Omega$. Если $\sigma(x) \equiv 0$ на $\partial \Omega$, то краевые условия будем называть, как обычно, условиями второго рода. Всюду в работе, если не оговорено противное, заданные функции в соотношениях (2.1)-(2.3) удовлетворяют условиям

$$
\begin{array}{ll}
\text { (A) } \quad \mu \in \mathrm{BV}[0, T], \quad \mu(0)=\mu(0+), \quad \mu(t) \not \equiv \text { const } \quad \text { на отрезке }[0, T], \\
& \rho(x, t) \geqslant \rho_{0}>0, \quad(x, t) \in Q ; \\
& \widetilde{g}, \widetilde{g}_{t} \in L_{2}(Q), \quad \widetilde{h}, \widetilde{h}_{t} \in L_{\infty, 2}(Q), \quad \widetilde{u}_{0} \in W_{2}^{2}(\Omega),
\end{array}
$$

(B) $\quad \exists \Phi(x, t): \Phi, \Phi_{t} \in W_{2}^{2,1}(Q), \quad$ причем

$$
\Phi(x, 0)=\widetilde{u}_{0}(x), \quad x \in \Omega, \quad B \Phi(x, t)=\widetilde{\beta}(x, t), \quad(x, t) \in S ;
$$

(C) $\quad \tilde{\chi} \in W_{2}^{2}(\Omega), \quad B \widetilde{\chi}(x)=l(\widetilde{\beta})(x), \quad x \in \partial \Omega, \quad|l(\widetilde{h})(x)| \geqslant \delta>0, \quad x \in \Omega$.

Здесь $\rho_{0}$ и $\delta$ - постоянные.

ОПРЕДЕЛЕниЕ 1. Обобщенным решением обратной задачи называется пара функций $\{\widetilde{u}(x, t) ; \widetilde{f}(x)\}, \widetilde{u} \in W_{2}^{2,1}(Q), \widetilde{f} \in L_{2}(\Omega)$, удовлетворяющая всем соотношениям в $(2.1)-(2.3)$. 
Обозначим через $u^{0}(x, t)$ решение прямой задачи $(2.1)-(2.2)$ с $\widetilde{f}(x) \equiv 0$. Такое решение существует, единственно и принадлежит $W_{2}^{2,1}(Q)[21 ;$ гл. $4, \S 9$ и гл. $3, \S 6]$. В силу условий $(\mathrm{A}),(\mathrm{B})$, наложенных на коэффициенты уравнения (2.1) и данные прямой задачи, это решение будет обладать дополнительной гладкостью, а именно $u_{t}^{0} \in C\left([0, T] ; L_{2}(\Omega)\right), u^{0} \in C\left([0, T] ; W_{2}^{2}(\Omega)\right)$ (см. 33$)$. Рассмотрим функции

$$
u(x, t)=\widetilde{u}(x, t)-u^{0}(x, t), \quad f(x)=l(\widetilde{h}) \widetilde{f}(x), \quad h(x, t)=\widetilde{h}(x, t) l(\widetilde{h})^{-1} .
$$

Тогда $\widetilde{h}(x, t) \widetilde{f}(x) \equiv h(x, t) f(x)$ в $Q$. По условию $(\mathrm{C})$ выполняется неравенство $|l(\widetilde{h})(x)| \geqslant \delta>0, x \in \Omega$, и, значит, оператор умножения на функцию $l(\widetilde{h})(x)$ непрерывно обратим в $L_{2}(\Omega)$. Поэтому обратная задача $(2.1)-(2.3)$ эквивалентно сводится к задаче о нахождении пары функций $\{u(x, t) ; f(x)\}$ из условий

$$
\begin{gathered}
\rho(x, t) u_{t}(x, t)-L(t) u(x, t)=h(x, t) f(x), \quad(x, t) \in Q, \\
u(x, 0)=0, \quad x \in \Omega, \quad B u(x, t)=0, \quad(x, t) \in S, \\
l(u)=\chi(x), \quad x \in \Omega,
\end{gathered}
$$

где $h(x, t) \equiv \widetilde{h}(x, t) l(\widetilde{h})^{-1}$ и $\chi(x) \equiv \widetilde{\chi}(x)-l\left(u^{0}\right)(x)$. Функции $\chi(x)$ и $h(x, t)$ будут удовлетворять условию

$$
\begin{gathered}
\chi \in W_{2}^{2}(\Omega), \quad B \chi(x)=0, \quad x \in \partial \Omega, \\
h, h_{t} \in L_{\infty, 2}(Q), \quad l(h)(x) \equiv 1, \quad x \in \Omega .
\end{gathered}
$$

Итак, задача (2.1)-(2.3) с условиями (А), (В), (C) может быть всегда сведена к задаче (2.4)-(2.6) с условиями (A), (D).

ЗАмечание 1. Решение прямой задачи (2.4)-(2.5) с фиксированным элементом $f \in L_{2}(\Omega)$ будем обозначать через $u(x, t ; f)$. В силу соответствующих теорем разрешимости (см. [21; гл. $4, \S 9$ и гл. $3, \S 6]$ ) такое решение существует и единственно в классе $W_{2}^{2,1}(Q)$, обладает дополнительной гладкостью, а именно $\forall f \in L_{2}(\Omega)$ выполнены включения $u_{t} \in C\left([0, T] ; L_{2}(\Omega)\right), u \in C\left([0, T] ; W_{2}^{2}(\Omega)\right)$ и $u_{t} \in W_{2}^{2,1}\left(Q_{\varepsilon}\right)$, где $Q_{\varepsilon}=\Omega \times(\varepsilon, T)$, при любом $\varepsilon \in(0, T)$ (подробнее см. $\S 3$ ). Поэтому для таких решений получаем, что $l(u) \equiv \int_{0}^{T} u(x, t) d \mu(t) \in W_{2}^{2}(\Omega)$. Это поясняет вопрос о требуемой гладкости функции $\chi(x)$ в условии $(2.6)$, поскольку априори для решения $u \in W_{2}^{2,1}(Q)$ можно гарантировать лишь включение $l(u) \in W_{2}^{1}(\Omega)$. В силу же большей гладкости по $t$ функции $h(x, t)$ и коэффициентов уравнения будем иметь более гладкое решение $u(x, t ; f)$ такое, что $l(u) \in W_{2}^{2}(\Omega)$. Таким образом, классы гладкости решения и заданных функций согласуются.

2.2. Описание результатов. В $\S 3$ для удобства ссылок приведены вспомогательные утверждения, используемые в работе. Это свойства, описывающие повышенную гладкость решения прямой задачи, а также свойства, связанные с неотрицательностью решений параболических уравнений.

В §4 даны формулировка и доказательство теоремы об эквивалентности обратной задачи (2.1)-(2.3) операторному уравнению второго рода относительно неизвестной функции $f(x)$ (см. теорему 1$)$. 
Для ряда последующих результатов будем предполагать, что в оператоpe $L(t)$ можно выделить стационарную часть $L_{0}$ :

$$
\begin{gathered}
L(t) u=L_{0} u+d(x, t) u, \\
L_{0} u=\sum_{i, j=1}^{n} \frac{\partial}{\partial x_{i}}\left(a_{i j}(x) \frac{\partial u}{\partial x_{j}}\right)+\sum_{i=1}^{n} b_{i}(x) \frac{\partial u}{\partial x_{i}}+c(x) u
\end{gathered}
$$

где коэффициенты $a_{i j}, b_{i}, d$ удовлетворяют условию $(\mathrm{A})$, функции $b_{i}$ теперь не зависят от $t$, а коэффициент $c(x)$ подчинен требованиям

$$
c \in L_{\infty}(\Omega), \quad c(x) \leqslant 0, \quad x \in \Omega
$$

причем, если $B u \equiv \frac{\partial u}{\partial N}, \quad$ то $c(x) \not \equiv 0 \quad$ в $\Omega$.

Такое выделение слагаемого с $c(x) \not \equiv 0$ существенно лишь для доказательства теоремы о корректности задачи с граничными условиями второго рода. Во всех остальных случаях можно считать, что $c(x) \equiv 0$ в $\Omega$.

В $\S 5$ даны достаточные условия корректности обратной задачи $(2.1)-(2.3)$ (см. теорему 2). Исследованию достаточных условий единственности решения обратной задачи посвящен $\S 6$ (теорема 3 ). Отдельно рассмотрена единственность решения для второй краевой задачи (теорема 4). В качестве следствия фредгольмовости второй краевой задачи из единственности получена корректность (теорема 5). Отметим, что в силу линейности задачи, ее единственность стандартным способом сводится к отсутствию ненулевых решений у однородной обратной задачи, т.е. задачи $(2.1)-(2.3)$ с функциями $\widetilde{g}=\widetilde{u}_{0}=\widetilde{\beta}=\widetilde{\chi}=0$. В $\S 7$ при определенных условиях на функцию $\widetilde{h}(x, t)$ дано описание ядра обратной задачи, т.е. множества пар $\{\widetilde{u}(x, t) ; \widetilde{f}(x)\}$, являющихся решениями однородной обратной задачи. В $\S 8$ изучена связь единственности и корректности обратной задачи с полнотой и базисностью в $L_{2}(\Omega)$ некоторой системы функций.

\section{§ 3. Вспомогательные утверждения}

3.1. Априорные оценки для доказательства фредгольмовости. Обозначим через $E$ вещественное банахово пространство, $\mathscr{L}(E)$ - пространство линейных ограниченных операторов в $E$ с операторной нормой, $\sigma(E)$ подпространство $\mathscr{L}(E)$ компактных операторов. Для удобства ссылок и полноты изложения приведем одно свойство интеграла от операторной функции. Близкие рассуждения использовались в работах [6], [9], [10].

Лемма 1. Пусть бункиия $\mu \in \mathrm{BV}[0, T] u \mu(0)=\mu(0+)$, а семейство операторов $\mathrm{G}(t):[0, T] \rightarrow \mathscr{L}(E)$ удовлетворяет условиям:

1. $\mathrm{G}(t) \in C((0, T] ; \mathscr{L}(E)) u \forall f \in E \quad\|[\mathrm{G}(t)-\mathrm{G}(0)] f\|_{E} \rightarrow 0$ npu $t \rightarrow 0+;$

2. $\forall t \in(0, T] \quad \mathrm{G}(t) \in \sigma(E)$.

Тогда определен линейный оператор $\mathbf{B}: \forall f \in E \mathbf{B} f:=\int_{0}^{T} \mathrm{G}(t) f d \mu(t)$, причем $\mathbf{B} \in \sigma(E)$. 
Действительно, по условию $\forall f \in E \mathrm{G}(t) f \in C([0, T] ; E)$, поэтому, используя принцип равномерной ограниченности (см., например, [22; гл. $2, \S 1]$ )

$$
\|\mathbf{B} f\|_{E} \leqslant \max _{t \in[0, T]}\left\{\|\mathrm{G}(t) f\|_{E}\right\} \bigvee_{0}^{T}(\mu) \leqslant C_{*} \bigvee_{0}^{T}(\mu)\|f\|_{E}, \quad \text { т.e. } \mathbf{B} \in \mathscr{L}(E) .
$$

Для любого $\delta \in(0, T)$ оператор $\mathbf{B}_{\delta}$ определим по правилу $\mathbf{B}_{\delta}:=$ $\int_{\delta}^{T} \mathrm{G}(t) d \mu(t)$. Имеем $\mathbf{B}_{\delta} \in \sigma(E)$, поскольку операторная функция $\mathrm{G}(t) \in$ $C([\delta, T] ; \mathscr{L}(E))$ и при каждом $t \in[\delta, T]$ оператор $\mathrm{G}(t) \in \sigma(E)$. Тогда

$$
\left\|\left(\mathbf{B}-\mathbf{B}_{\delta}\right) f\right\|_{E} \leqslant \max \left\{\|\mathrm{G}(t) f\|_{E} \mid t \in[0, \delta]\right\} \bigvee_{0}^{\delta}(\mu) \leqslant C_{*}\|f\|_{E} \bigvee_{0}^{\delta}(\mu) \rightarrow 0
$$

при $\delta \rightarrow 0+($ см. [23; гл. 8, $\S 1$, теорема 1$])$. Множество $\sigma(E)$ замкнуто в норме $\mathscr{L}(E)$, поэтому $\mathbf{B} \in \sigma(E)$.

Сейчас докажем несколько утверждений о свойствах гладкости решения прямой задачи. Фиксируем $f \in L_{2}(\Omega)$ и введем функцию $u(x, t ; f)$ как решение прямой задачи

$$
\begin{gathered}
\rho(x, t) u_{t}(x, t)-L(t) u(x, t)=h(x, t) f(x), \quad(x, t) \in Q, \\
u(x, 0)=0, \quad x \in \Omega, \quad B u(x, t)=0, \quad(x, t) \in S,
\end{gathered}
$$

где коэффициенты удовлетворяют условию $(\mathrm{A}), h, h_{t} \in L_{\infty, 2}(Q)$, а равномерно эллиптический оператор $L(t)$ и оператор краевых условий $B$ такие же, как в задаче $(2.1)-(2.3)$. Известно (см. [21; гл. $4, \S 9$ и гл. $3, \S 6])$, что у задачи $(3.1)$, (3.2) существует единственное решение $u \in W_{2}^{2,1}(Q)$. Для фиксированного решения $u(x, t ; f)$ рассмотрим еще задачу нахождения $w(x, t)$ из условий

$$
\begin{gathered}
\rho(x, t) w_{t}(x, t)-L(t) w(x, t)+\rho_{t} w=h_{t} f+L_{t}(t) u(x, t), \quad(x, t) \in Q, \\
w(x, 0)=h(x, 0) f(x)[\rho(x, 0)]^{-1}, \quad x \in \Omega, \quad B w(x, t)=0, \quad(x, t) \in S,
\end{gathered}
$$

где

$$
L_{t}(t) u:=\sum_{i, j=1}^{n} \frac{\partial}{\partial x_{i}}\left(\left(\partial_{t} a_{i j}\right) \frac{\partial u}{\partial x_{j}}\right)+\left(\vec{b}_{t}, \nabla u\right)+d_{t} u \equiv\left(\vec{b}_{t}, \nabla u\right)+d_{t} u
$$

- это продифференцированный оператор $L(t)$, начальное условие

$$
v_{0}(x ; f):=h(x, 0) f(x)[\rho(x, 0)]^{-1} \in L_{2}(\Omega),
$$

а правая часть $F(x, t ; f):=h_{t}(x, t) f(x)+L_{t}(t) u(x, t) \in L_{2}(Q)$. Задача $(3.3),(3.4)$ имеет единственное решение $w(x, t)$ в классе $V_{2}^{1,0}(Q)$, причем это решение (см. $[21 ;$ гл. $3, \S 4])$ удовлетворяет условию $w \in C\left([0, T] ; L_{2}(\Omega)\right)$. Отметим, что (3.3), (3.4) - это формально продифференцированная задача (3.1), (3.2).

ЛЕмма 2. При любом $f \in L_{2}(\Omega)$ решение задачи (3.1), (3.2) $u(x, t ; f) \in$ $W_{2}^{2,1}(Q)$ и дополнительно удовлетворяет следующим условиям: 
1. $u_{t}(\cdot, t ; f) \in C\left([0, T] ; L_{2}(\Omega)\right) u \forall t \in(0, T]$ справедлива оченка

$$
\left\|u_{t}(\cdot, t+\Delta t ; f)-u_{t}(\cdot, t ; f)\right\|_{2, \Omega} \leqslant \alpha_{0}(t, \Delta t)\|f\|_{2, \Omega},
$$

где положительная функиия $\alpha_{0}(t, \Delta t) \rightarrow 0$ при $\Delta t \rightarrow 0$;

2. $\forall t \in(0, T] \exists K(t)>0:\left\|u_{t}(\cdot, t ; f)\right\|_{2, \Omega}^{(1)} \leqslant K(t)\|f\|_{2, \Omega}$.

ДокАЗАтЕльство. Зафиксируем произвольную функцию $f(x) \in L_{2}(\Omega)$. Как уже отмечалось, для данной $f(x)$ существует единственная функция $u(x, t ; f)$, $u \in W_{2}^{2,1}(Q),-$ решение задачи (3.1), (3.2) (см. [21]). Обозначим $v(x, t ; f):=$ $u_{t}(x, t ; f) \in L_{2}(Q)$ и докажем, что $v$ является решением задачи $(3.3),(3.4)$ в классе $V_{2}(Q)$. Тогда в силу единственности оно совпадает с функцией $w \in$ $V_{2}^{1,0}(Q) \subset V_{2}(Q)$, которая также является решением задачи $(3.3),(3.4)$ в классе $V_{2}(Q)$. Для случая первой краевой задачи $w(x, t)$ удовлетворяет тождеству (см. [21; гл. $3, \S 1]$ )

$$
\begin{aligned}
\int_{\Omega} \rho(x, t) & w(x, t) \eta(x, t) d x-\int_{0}^{t} \int_{\Omega} \rho w \eta_{t} d x d t \\
& \quad+\int_{0}^{t} \int_{\Omega}\left[a_{i j} w_{x_{j}} \eta_{x_{i}}-(\vec{b}, \nabla w) \eta-\left(d+\rho_{t}\right) w \eta\right] d x d t \\
= & \int_{0}^{t} \int_{\Omega} F(x, t) \eta(x, t) d x d t+\int_{\Omega} \rho(x, 0) w(x, 0) \eta(x, 0) d x
\end{aligned}
$$

с любой функцией $\eta(x, t) \in W_{2}^{1,1}(Q)$ такой, что $\eta(x, t)=0,(x, t) \in S$, где $w(x, 0)=v_{0}(x ; f)$. Для случая третьей (второй) краевой задачи $w(x, t)$ удовлетворяет с любой функцией $\eta(x, t) \in W_{2}^{1,1}(Q)$ такому тождеству:

$$
\begin{aligned}
\int_{\Omega} \rho(x, t) & w(x, t) \eta(x, t) d x-\int_{0}^{t} \int_{\Omega} \rho w \eta_{t} d x d t \\
& \quad+\int_{0}^{t} \int_{\Omega}\left[a_{i j} w_{x_{j}} \eta_{x_{i}}-(\vec{b}, \nabla w) \eta-\left(d+\rho_{t}\right) w \eta\right] d x d t+\int_{0}^{t} \int_{\partial \Omega} \sigma w \eta d s d t \\
= & \int_{0}^{t} \int_{\Omega} F(x, t) \eta(x, t) d x d t+\int_{\Omega} \rho(x, 0) w(x, 0) \eta(x, 0) d x,
\end{aligned}
$$

где по повторяющимся индексам $i, j$ производится суммирование от 1 до $n$.

Для функции $u(x, t ; f)$, в силу дополнительной гладкости по $t$ коэффициентов и правой части, методами работ [21] и [24] может быть получена априорная оценка нормы $u_{t}$ в пространстве $V_{2}(Q)$. После этого уже стандартным рассуждением выводим, что $v=u_{t}$ удовлетворяет одному из приведенных выше тождеств, т.е. является решением задачи $(3.3),(3.4)$ в классе $V_{2}(Q)$, и, следовательно, $v$ совпадает с $w \in V_{2}^{1,0}(Q)$. Поэтому $u_{t}(\cdot, t ; f) \in C\left([0, T] ; L_{2}(\Omega)\right)($ см. $[21 ;$ гл. $3, \S 4])$.

Как обозначено выше, $F(x, t ; f)=h_{t} f+L_{t} u$ - это правая часть (3.3). Тогда $\|F\|_{2, Q} \leqslant C_{1}\|f\|_{2, \Omega}+C_{2}\|u\|_{2, Q}^{(2,1)} \leqslant C_{3}\|f\|_{2, \Omega}$ в силу оценки нормы решения $u(x, t ; f)$ в пространстве $W_{2}^{2,1}(Q)$. Зафиксируем произвольное $\varepsilon \in(0, T)$ и определим функцию $\eta(\tau) \in C^{2}([0, T])$ так, чтобы $\eta(0)=0, \eta(\tau)=1$ при $\tau \in[\varepsilon, T]$ и $0 \leqslant \eta(\tau) \leqslant 1$ при $\tau \in[0, T]$. Введем функцию $\widetilde{w}(x, t):=\eta(t) v(x, t ; f) \in V_{2}^{1,0}(Q)$. 
Тогда $\widetilde{w}(x, t)$ - это решение следующей задачи (в классе $\left.V_{2}^{1,0}(Q)\right)$ :

$$
\begin{gathered}
\rho \widetilde{w}_{t}-L(t) \widetilde{w}+\rho_{t} \widetilde{w}=h_{t} \eta(t) f+L_{t}(t)(\eta u)+\rho \eta_{t} v, \quad(x, t) \in Q, \\
\widetilde{w}(x, 0)=0, \quad x \in \Omega, \quad B \widetilde{w}(x, t)=0, \quad(x, t) \in S .
\end{gathered}
$$

Обозначим $F_{1}(x, t ; f, \eta):=h_{t} \eta(t) f+L_{t}(t)(\eta u)+\rho \eta_{t} v \in L_{2}(Q)$. У задачи (3.5), (3.6) существует и притом единственное решение в классе $W_{2}^{2,1}(Q)$, которое также является решением в $V_{2}^{1,0}(Q)$. Из единственности решения в $V_{2}^{1,0}(Q)$ имеем, что $\widetilde{w} \in W_{2}^{2,1}(Q)$. В силу оценки устойчивости получим

$$
\|\widetilde{w}\|_{2, Q}^{(2,1)} \leqslant C_{1}\left\|F_{1}\right\|_{2, Q} \leqslant C_{2}\left(\eta, \eta_{t}\right)\|f\|_{2, \Omega}
$$

Функция $\widetilde{w}(x, t)$ при $t \in[0, T]$ имеет след из $W_{2}^{1}(\Omega)$, поэтому для всех $t \in[0, T]$ справедливо неравенство $\|\widetilde{w}(\cdot, t)\|_{2, \Omega}^{(1)} \leqslant C_{3}(t)\|\widetilde{w}\|_{2, Q}^{(2,1)}$. Возьмем произвольное $t \in(0, T]$, фиксируем его, выберем $\varepsilon=t / 2$ и построим функцию $\eta$, как указано выше. Тогда существует $K(t)>0$ :

$$
\|\widetilde{w}(\cdot, t)\|_{2, \Omega}^{(1)}=\left\|u_{t}(\cdot, t ; f)\right\|_{2, \Omega}^{(1)} \leqslant K(t)\|f\|_{2, \Omega} .
$$

Здесь учтено, что $t>\varepsilon$ и для него $\eta(t)=1$. Оценка п. 2 леммы 2 доказана.

Докажем оценку из п. 1. Рассмотрим $\varepsilon>0$. Тогда для $t, t+\Delta t \in(\varepsilon, T]$ имеем

$$
|\widetilde{w}(x, t+\Delta t)-\widetilde{w}(x, t)| \leqslant\left|\int_{t}^{t+\Delta t}\right| \widetilde{w}_{\tau}(x, \tau)|d \tau|
$$

для почти всех $x \in \Omega$. Отсюда по неравенству Коши-Буняковского

$$
\int_{\Omega}|\widetilde{w}(x, t+\Delta t)-\widetilde{w}(x, t)|^{2} d x \leqslant\left.|\Delta t|\left|\int_{t}^{t+\Delta t} \int_{\Omega}\right| \widetilde{w}_{\tau}(x, \tau)\right|^{2} d \tau \mid,
$$

и для фиксированного $t \in(0, T]$ получим неравенство

$$
\left\|u_{t}(x, t+\Delta t ; f)-u_{t}(x, t ; f)\right\|_{2, \Omega} \leqslant|\Delta t|^{1 / 2} C(t)\|f\|_{2, \Omega} .
$$

Здесь мы воспользовались оценкой (3.7). Лемма доказана.

СледСтвиЕ 1. Семейство операторов $\mathrm{G}_{1}(t) f:=\rho u_{t}(\cdot, t ; f)$ удовлетворяет условиям леммы 1.

ДоказАТЕЛЬСтво. Оператор умножения на функцию $\rho(x, t)$ при любом значении $t \in[0, T]$ ограничен из $L_{2}(\Omega)$ в $L_{2}(\Omega)$ и непрерывен по $t \in[0, T]$ в норме $\mathscr{L}(E)$, где $E=L_{2}(\Omega)$. Операторное семейство $\mathrm{G}_{2}(t) f:=u_{t}(\cdot, t ; f)$ удовлетворяет условиям леммы 1 , так как компактность $\mathrm{G}_{2}(t)$ следует из оценки (3.8) и теоремы Реллиха-Кондрашова. Отсюда получим, что и $\mathrm{G}_{1}(t)$ удовлетворяет условиям леммы 1. Следствие доказано.

ЛЕмма 3. При любом $f \in L_{2}(\Omega)$ решение задачи $(3.1),(3.2) u(x, t ; f)$ удовлетворяет следующим условиям:

1. $u(\cdot, t ; f) \in C\left([0, T] ; W_{2}^{2}(\Omega)\right)$; 
2. $\forall i=\overline{1, n}$ справедливо включение $u_{x_{i}}(\cdot, t ; f) \in C\left([0, T] ; W_{2}^{1}(\Omega)\right)$ и существует такая постоянная $C_{*}>0$, что для всех $t \in[0, T]$ выполнено неравенство

$$
\left\|u_{x_{i}}(\cdot, t ; f)\right\|_{2, \Omega}^{(1)} \leqslant\|u(\cdot, t ; f)\|_{2, \Omega}^{(2)} \leqslant C_{*}\|f\|_{2, \Omega}
$$

3. $\forall t, t+\Delta t \in[0, T]$ имеет место оценка

$$
\left\|u_{x_{i}}(\cdot, t+\Delta t ; f)-u_{x_{i}}(\cdot, t ; f)\right\|_{2, \Omega} \leqslant \alpha_{1}(t, \Delta t)\|f\|_{2, \Omega},
$$

где положительная функиия $\alpha_{1}(t, \Delta t) \rightarrow 0$ при $\Delta t \rightarrow 0$.

ДокАЗАТЕЛЬСтво. Используя следствие 1 и условия $(\mathrm{A}),(\mathrm{D})$, получим, что $\rho(\cdot, t) u_{t}(\cdot, t ; f) \in C\left([0, T] ; L_{2}(\Omega)\right)$ при любом $f \in L_{2}(\Omega)$. Тогда из уравнения (3.1) имеем равенство

$$
-L(t) u=h(x, t) f(x)-\rho(x, t) u_{t}(x, t),
$$

правую часть которого обозначим $\gamma(x, t):=h(x, t) f(x)-\rho(x, t) u_{t}(x, t ; f)$. Без ограничения общности будем считать, что $d(x, t) \leqslant d_{0}<0$ в $Q$ с некоторой константой $d_{0}$. Функция $\gamma(x, t)$ непрерывно меняется в норме $L_{2}(\Omega)$ на $[0, T]$. Рассмотрим уравнение (3.9) при $t, t+\Delta t \in[0, T]$ и вычтем одно из другого, обозначив

$\delta u:=u(x, t+\Delta t)-u(x, t), \quad \delta \gamma:=\gamma(x, t+\Delta t)-\gamma(x, t), \quad \delta L:=L(t+\Delta t)-L(t)$.

Отсюда выводим при $t, t+\Delta t \in[0, T]$ равенство в области $\Omega$

$$
-L(t+\Delta t) \delta u=(\delta L) u+\delta \gamma
$$

Из второго основного неравенства для эллиптических операторов [25; гл. $3, \S 8]$ и неравенства треугольника имеем оценку

$$
\|\delta u\|_{2, \Omega}^{(2)} \leqslant C\left(\|(\delta L) u\|_{2, \Omega}+\|\delta \gamma\|_{2, \Omega}\right) .
$$

Правая часть (3.10) стремится к нулю при $\Delta t \rightarrow 0$, так как

$$
\gamma(\cdot, t) \in C\left([0, T] ; L_{2}(\Omega)\right), \quad\|(\delta L) u\|_{2, \Omega} \leqslant \alpha(t, \Delta t)\|u(\cdot, t)\|_{2, \Omega}^{(1)},
$$

где функция $\alpha(t, \Delta t) \rightarrow 0$ при $\Delta t \rightarrow 0$. Здесь учтено, что старшие коэффициенты эллиптического оператора $L$ не зависят от $t$. Таким образом, из (3.10) имеем, что $u(\cdot, t ; f) \in C\left([0, T] ; W_{2}^{2}(\Omega)\right)$. В силу равенства $(3.9)$ и второго основного неравенства для эллиптических операторов [25; гл. $3, \S 8]$

$$
\|u(\cdot, t ; f)\|_{2, \Omega}^{(2)} \leqslant C\|L(t) u\|_{2, \Omega}=C\left\|h f-\rho \cdot u_{t}\right\|_{2, \Omega} \leqslant C_{1}\|f\|_{2, \Omega},
$$

так как для функции $v=u_{t}$ как решения задачи $(3.3),(3.4)$ справедлива оценка "энергетической нормы” [21; гл. $3, \S 2]$

$$
\max _{[0, T]}\|v(\cdot, t)\|_{2, \Omega}+\left\|v_{x}\right\|_{2, Q} \leqslant C\left(\left\|v_{0}\right\|_{2, \Omega}+\|F\|_{2,1, Q}\right) \leqslant C_{1}\|f\|_{2, \Omega},
$$


где $v_{0}(x ; f)=h(x, 0) f(x) / \rho(x, 0), F(x, t ; f)=h_{t} f+L_{t} u, u=u(x, t ; f)$ - решение задачи (3.1), (3.2). Отсюда для всех $i=\overline{1, n}$ имеем искомую оценку

$$
\left\|u_{x_{i}}(\cdot, t ; f)\right\|_{2, \Omega}^{(1)} \leqslant\|u(\cdot, t ; f)\|_{2, \Omega}^{(2)} \leqslant C_{*}\|f\|_{2, \Omega} \quad \forall t \in[0, T] .
$$

Из (3.11) и неравенства Коши-Буняковского с учетом $u_{t x_{i}} \in L_{2}(Q)$ следует, что

$$
\left\|u_{x_{i}}(\cdot, t+\Delta t ; f)-u_{x_{i}}(\cdot, t ; f)\right\|_{2, \Omega} \leqslant C|\Delta t|^{1 / 2}\|f\|_{2, \Omega},
$$

и лемма 3 доказана.

СЛЕДСТвИЕ 2. При любом значении $i=\overline{1, n}$ семейство операторов $\mathrm{G}_{i}(t)$, задаваемое равенством $\mathrm{G}_{i}(t) f:=b_{i}(\cdot, t) u_{x_{i}}(\cdot, t ; f)$, удовлетворяет условиям леммы 1.

ДокАЗАТЕльство аналогично доказательству следствия 1.

Лемма 4. При любом $f \in L_{2}(\Omega)$ решение задачи $(3.1),(3.2) u(x, t ; f)$ удовлетворяет следующим условиям:

1. $\forall t, t+\Delta t \in[0, T]$ имеет место оценка

$$
\|u(\cdot, t+\Delta t ; f)-u(\cdot, t ; f)\|_{2, \Omega} \leqslant \alpha(t, \Delta t)\|f\|_{2, \Omega},
$$

где положительная функиия $\alpha(t, \Delta t) \rightarrow 0$ при $\Delta t \rightarrow 0$;

2. $\forall t \in[0, T] \exists K(t)>0:\|u(\cdot, t ; f)\|_{2, \Omega}^{(1)} \leqslant K(t)\|f\|_{2, \Omega}$.

ДокАЗАТЕЛЬСтво следует из включения $u \in W_{2}^{2,1}(Q)$ и оценки решения $\|u\|_{2, Q}^{(2,1)} \leqslant C\|f\|_{2, \Omega}$.

СлеДСтвиЕ 3. Семейство операторов $\mathrm{G}_{d}(t)$ удовлетворяет условиям леммы 1, где $\mathrm{G}_{d}(t) f:=d(\cdot, t) u(\cdot, t ; f)$.

3.2. О положительных решениях параболических уравнений. Здесь для удобства ссылок дадим ряд утверждений, связанных со свойствами положительных решений параболических уравнений. Пусть всюду далее в этом параграфе выполняются условия

$$
F \in L_{2}(Q), u_{0} \in L_{2}(\Omega), \quad F(x, t) \geqslant 0,(x, t) \in Q, \quad u_{0}(x) \geqslant 0, x \in \Omega,
$$

а функция $u(x, t) \in V_{2}^{1,0}(Q)$ (см. [21; гл. $\left.\left.3, \S \S 4,5\right]\right)$ - решение задачи

$$
\begin{gathered}
\rho(x, t) u_{t}(x, t)-L(t) u(x, t)=F(x, t), \quad(x, t) \in Q, \\
u(x, 0)=u_{0}(x), \quad x \in \Omega, \quad B u(x, t)=0, \quad(x, t) \in S,
\end{gathered}
$$

где $\rho(x, t)$ и коэффициенты $L(t)$ удовлетворяют условию $(\mathrm{A})$, при этом считаем, что старшие коэффициенты $a_{i j}=a_{i j}(x, t) \in C^{1}(\bar{Q})$. Оператор краевых условий $B$ имеет вид

$$
\text { либо } B u=u, \quad \text { либо } B u=\frac{\partial u}{\partial N}+\sigma(x, t) u,
$$

где

$$
\frac{\partial u}{\partial N} \equiv \sum_{i, j=1}^{n} \cos \left(\mathbf{n}, x_{i}\right) a_{i j}(x, t) \frac{\partial u}{\partial x_{j}}
$$


$\mathbf{n}$ - внешняя нормаль к $\partial \Omega$ в точке $x=\left(x_{1}, \ldots, x_{n}\right), \sigma \in C^{1}(S), \sigma(x, t) \geqslant 0$ на $S$. Так как коэффициент $d(x, t)$ ограничен, то без потери общности считаем в этом пункте, что $d(x, t) \leqslant 0$ в $Q$.

Приведем соответствующие результаты о неотрицательности решения задачи (3.13), (3.14).

Лемма 5. Пусть выполнены условия (3.12), а и(x,t) - решение задачи (3.13), (3.14). Тогда функиия и $(x, t)$ обладает следующими свойствами:

1. $u(x, t) \geqslant 0$ в $Q$;

2. если $u_{0}(x) \not \equiv 0$ в $\Omega$, mо $\forall t \in(0, T] \quad u(x, t)>0$ в $\Omega$;

3. если $u_{0}(x) \equiv 0$ в $\Omega$, а $F(x, t) \not \equiv 0$ в $Q_{t_{0}}:=\Omega \times\left(0, t_{0}\right)$ для некоторого $t_{0} \in(0, T], m o \forall t \in\left[t_{0}, T\right] \quad u(x, t)>0$ в $\Omega$;

4. если $u_{0}(x) \equiv 0$ в $\Omega, F(\cdot, t) \in C\left([0, T] ; L_{p}(\Omega)\right)$ при $p \geqslant 1$ и $F\left(x, t_{0}\right) \not \equiv 0$ в $\Omega$ для некоторого $t_{0} \in(0, T]$, mo $\forall t \in\left[t_{0}, T\right] u(x, t)>0$ в $\Omega$.

ДоказАтельство. Так как $F \in L_{2}(Q), u_{0} \in L_{2}(\Omega)$, то существует единственное решение $u \in V_{2}^{1,0}(Q)$ (см. $[21 ;$ гл. $\left.3, \S \S 4,5]\right)$, причем функция $u(x, t)$ непрерывна на $[0, T]$ в норме $L_{2}(\Omega)$. Применяя слабый принцип максимума для решений параболических уравнений в $V_{2}^{1,0}(Q)$ (см., например, [24; гл. 4, $\S \S 7,10])$, получим $u(x, t) \geqslant 0$ в $Q$, т.е. выполнено свойство 1 . Таким образом, $u(x, t)$ - неотрицательное суперрешение параболического уравнения.

Введем в рассмотрение функцию $v \in V_{2}^{1,0}(Q)$ как решение задачи (3.13), (3.14) с $F(x, t) \equiv 0$ в $Q$. Из слабого принципа максимума для параболических уравнений имеем $u(x, t) \geqslant v(x, t) \geqslant 0$ в $Q$. Так как $u_{0}(x) \geqslant 0$ в $\Omega$, то найдется монотонно возрастающая последовательность непрерывных функций $\left\{u_{0}^{k}(x)\right\}$, сходящаяся п.в. в $\Omega$ к $u_{0}(x)$ (см. [26; гл. $\left.\left.2, \S 1\right]\right)$. Обозначив $v^{k}(x, t)$ решение задачи $(3.13),(3.14)$ с правой частью $F=0$ и начальным условием $u_{0}(x)=$ $u_{0}^{k}(x)$, имеем $v^{k} \in W_{p}^{2,1}\left(Q_{\varepsilon}\right) \cap V_{2}^{1,0}(Q)$ с $p \geqslant n+1$, и поэтому $v^{k} \in C\left(\bar{Q}_{\varepsilon}\right)$, где $Q_{\varepsilon}=\Omega \times(\varepsilon, T), \varepsilon \in(0, T)$.

Из оценки устойчивости решения в $V_{2}^{1,0}(Q)$ получим, что для всех $t \in[0, T]$ последовательность $v^{k}(x, t)$ сходится к $v(x, t)$ в норме $L_{2}(\Omega)$. В силу слабого принципа максимума $v(x, t) \geqslant v^{k+1}(x, t) \geqslant v^{k}(x, t)$ в $Q$. Фиксируем $t \in(0, T]$ и возьмем $\varepsilon=t / 2$. Без ограничения общности считаем, что $u_{0}^{k}(x) \not \equiv 0$ в $\Omega$ при всех $k \in \mathbb{N}$. Применяя строгий принцип максимума для непрерывных обобщенных решений параболических уравнений, получим, что $v^{k}(x, t)>0$ в $\Omega$. Так как существует подпоследовательность $v^{k}(x, t)$, сходящаяся п.в. в $\Omega$ к $v(x, t)$, то $u(x, t) \geqslant v(x, t)>0$ в $\Omega$, и свойство 2 леммы установлено. Отметим, что строгий принцип максимума для непрерывных обобщенных решений выводится из слабого неравенства Харнака для неотрицательных суперрешений параболических уравнений (см., например, [24; гл. $4, \S \S 7,10],[27])$.

Предположим теперь, что $u_{0}(x) \equiv 0$ в $\Omega$, а $F(x, t) \not \equiv 0$ в $Q_{t_{0}}:=\Omega \times\left(0, t_{0}\right)$. Так как $F(x, t) \geqslant 0$ в $Q$ и $F \in L_{2}(Q)$, то найдется монотонно возрастающая последовательность непрерывных функций $\left\{F^{k}(x, t)\right\}$, сходящаяся п.в. в $Q$ к $F(x, t)$. Будем считать, что $F^{k}(x, t) \not \equiv 0$ в $Q_{t_{0}} \subseteq Q$ для всех $k \in \mathbb{N}$. Так как $F^{k}(x, t) \rightarrow F(x, t)$ в $L_{2}(Q)$ при $k \rightarrow \infty$, то из оценки устойчивости в $V_{2}^{1,0}(Q)$ имеем $u^{k}(x, t) \rightarrow u(x, t)$ в $L_{2}(\Omega)$ при $k \rightarrow \infty$ для каждого $t \in[0, T]$. Здесь $u^{k}(x, t)-$ решение задачи $(3.13),(3.14)$ с правой частью $F=F^{k}$ и начальным условием 
$u_{0}=0$. Отметим, что $u^{k} \in W_{p}^{2,1}(Q)$ с $p \geqslant n+1$, так как $F_{k} \in L_{p}(Q)$, и поэтому $u^{k} \in C(\bar{Q})$, причем последовательность $\left\{u^{k}(x, t)\right\}$ монотонно возрастает.

Рассмотрим произвольное $t_{*} \in\left[t_{0}, T\right]$. При всех $k \in \mathbb{N}$ в области $\Omega$ справедливо неравенство $u^{k}\left(x, t_{*}\right)>0$. Действительно, в противном случае из строгого принципа максимума получили бы $u^{k}(x, t) \equiv 0$ в $\bar{Q}_{t_{*}}:=\bar{\Omega} \times\left[0, t_{*}\right]$ и, следовательно, $F^{k}(x, t) \equiv 0$ в $\bar{Q}_{t_{*}}$, что противоречит условию $F^{k}(x, t) \not \equiv 0$ в $Q_{t_{0}}$. Используя оценку устойчивости в $V_{2}^{1,0}(Q)$ и переходя, если необходимо, к подпоследовательности, при каждом $t \in\left[t_{0}, T\right]$ получим, что $u^{k}(x, t) \rightarrow u(x, t)>0$ п.в. в $\Omega$, т.е. свойство 3 доказано. Из условий последнего пункта леммы вытекает, что $F(x, t) \not \equiv 0$ в $Q_{t_{0}}$, и поэтому свойство 4 следует из свойства 3. Лемма доказана.

Лемма 6. Пусть выполнены условия (3.12), а и(x,t)-решение прямой задачи $(3.13),(3.14)$. Тогда при любом $t_{0} \in(0, T]$ для функи,ии и $(x, t)$ справедливо одно из следующих утверждений:

1. либо $u\left(x, t_{0}\right) \equiv 0$ в $\Omega$, а тогда $u(x, t) \equiv 0{ }_{\text {в }} \bar{Q}_{t_{0}} u F(x, t) \equiv 0{ }_{\text {в }} \bar{Q}_{t_{0}}$;

2. либо $и\left(x, t_{0}\right)>0$ в $\Omega$, и тогда найдется $\delta \in\left(0, t_{0}\right)$ такое, что для всех $t \in\left[t_{0}-\delta, t_{0}\right]$ выполняется неравенство $u(x, t)>0$ в $\Omega$.

ДокАЗАТЕЛЬство. Возьмем произвольное $t_{0} \in(0, T]$ и фиксируем его. По лемме $5 u\left(x, t_{0}\right) \geqslant 0$ в $\Omega$. Если $u\left(x, t_{0}\right)=0$ на множестве $\Omega_{0} \subset \Omega$ с мерой $\left|\Omega_{0}\right|>0$, то по лемме 5 одновременно $u_{0}(x) \equiv 0$ в $\Omega$ и $F(x, t) \equiv 0$ в $\bar{Q}_{t_{0}}$. Но тогда в силу единственности решения прямой задачи имеем $u(x, t) \equiv 0$ в $\bar{Q}_{t_{0}}$, т.е. реализуется первый случай доказываемой альтернативы.

Если же $u\left(x, t_{0}\right)>0$ в $\Omega$, то в силу непрерывности $u(x, t)$ в норме $L_{2}(\Omega)$ на $[0, T]$ найдется $\delta \in\left(0, t_{0}\right)$ такое, что $\|u(\cdot, t)\|_{2, \Omega}>0$ для всех $t \in\left[t_{0}-\delta, t_{0}\right]$. Тогда $u(x, t)>0$ в $\Omega$ при тех же значениях $t$. Лемма доказана.

3.3. Строгая монотонность интеграла Римана-Стилтьеса. Отметим для удобства ссылок несколько свойств интеграла Римана-Стилтьеса от положительных функций. Относительно функции $\mu(t)$, входящей в условие переопределения (2.3), дополнительно к условию (А) будем предполагать, что

$\mu(t)$ является неубывающей, непрерывной справа на $[0, T], \bigvee_{0}^{T}(\mu)>0$,

причем для непрерывности функции $\mu(t)$ в точке $t=T$ продолжим ее значением $\mu(T)$ при всех $t \geqslant T$. Заметим, что дополнительное к $(\mathrm{A})$ условие непрерывности $\mu(t)$ справа при всех $t>0$ не является ограничением общности (см. [23; гл. 8, §7]).

ОПРЕДЕЛЕНИЕ 2. Пусть функция $\mu(t)$ удовлетворяет условию (3.15). Точку $t_{*} \in(0, T]$ будем называть точкой роста $\mu(t)$ слева, если для любого $\delta \in\left(0, t_{*}\right)$ выполняется неравенство $\bigvee_{t_{*}-\delta}^{t_{*}}(\mu)>0$.

Обозначим $\mathscr{M}=\left\{t_{*} \in(0, T] \mid t_{*}-\right.$ точка роста $\mu(t)$ слева $\}$. Заметим, что при выполнении (3.15) $\mathscr{M} \neq \varnothing$ и число $\tau:=\sup \mathscr{M}$ само является точкой роста функции $\mu(t)$ слева, т.е. $\tau \in \mathscr{M}$. Кроме того, правее $\tau$ на $[0, T]$ у функции $\mu(t)$ вообще нет точек роста, т.е. $\mu(t) \equiv$ const на $[\tau, T]$. В связи с этим $\tau$ будем называть крайней правой точкой роста функции $\mu(t)$. 
Лемма 7. Пусть скалярная функиия $\mu(t)$ удовлетворяет условиям (3.15) u $t_{*} \in(0, T]$ есть ее точка роста слева. Если функция $\eta(t) \in C([0, T]), \eta(t) \geqslant 0$ на $[0, T]$ и $\eta\left(t_{*}\right)>0$, тогда $l(\eta):=\int_{0}^{T} \eta(t) d \mu(t)>0$.

Действительно, функция $\eta(t)$ непрерывна, следовательно $\eta(t) \geqslant \eta\left(t_{*}\right) / 2$ для всех $t \in\left[t_{*}-\delta, t_{*}\right]$, где $\delta>0$ достаточно мало, поэтому справедлива цепочка неравенств

$$
\int_{0}^{T} \eta(t) d \mu(t) \geqslant \int_{t_{*}-\delta}^{t_{*}} \eta(t) d \mu(t) \geqslant \frac{1}{2} \eta\left(t_{*}\right) \bigvee_{t_{*}-\delta}^{t_{*}}(\mu)>0
$$

Отсюда получаем такие два утверждения.

СлЕДСТвИЕ 4. Пусть $\mu(t)$ удовлетворяет условиям (3.15) $u t_{*} \in(0, T]$ есть ее точка роста слева. Если для п.в. $x \in \Omega$ функиия и $(x, t)$ непреръвна по переменной $t \in[0, T]$ и удовлетворяет неравенствам $u(x, t) \geqslant 0$ в $Q$, $u\left(x, t_{*}\right)>0$ п.в. в $\Omega$, тогда $l(u)(x):=\int_{0}^{T} u(x, t) d \mu(t)>0$ п.в. в $\Omega$.

СлеДСТвиЕ 5. Пусть $\mu(t)$ удовлетворяет условиям (3.15), функиия $u(x, t)$ для п.в. $x \in \Omega$ непрерывна по переменной $t \in[0, T]$, удовлетворяет неравенству $u(x, t) \geqslant 0$ в $Q$, a $l(u)(x):=\int_{0}^{T} u(x, t) d \mu(t) \equiv 0$ в $\Omega$. Тогда для любого $t_{*} \in \mathscr{M}$ справедливо равенство $u\left(x, t_{*}\right) \equiv 0 u$, в частности, $u(x, \tau) \equiv 0$ в $\Omega$, где множество $\mathscr{M}$ и число $\tau$ введены в определении 2.

Приведенные утверждения позволяют получить важное для доказательства теорем единственности и разрешимости свойство.

ЛЕмма 8. Пусть и $(x, t)$ - решение задачи (3.13), (3.14) и выполнень условия $(3.12),(3.15)$, причем $F(\cdot, t) \in C\left([0, T] ; L_{p}(\Omega)\right)$ с некоторьм $p \geqslant 1$, а $\tau-$ крайняя правая точка роста функиии $\mu(t)$. Eсли $l(u)(x) \equiv 0$ в $\Omega$, то $u(x, t) \equiv 0$ ${ }_{\text {в }} \bar{Q}_{\tau}, F(x, t) \equiv 0$ в $\bar{Q}_{\tau}, l(F)(x):=\int_{0}^{T} F(x, t) d \mu(t) \equiv 0$ в $\Omega$. Если же $l(u)(x) \not \equiv 0$ в $\Omega, \operatorname{mol}(u)(x)>0$ в $\Omega$.

ДокаЗАТЕЛьство. Пусть $l(u) \equiv 0$ в $\Omega$. Применяя следствие 5 , получим $u(x, \tau) \equiv 0$ в $\Omega$, а тогда по лемме 6 имеем $u(x, t) \equiv 0$ и $F(x, t) \equiv 0$ в $\bar{Q}_{\tau}$. Функция $\mu(t)$ не имеет точек роста на $(\tau, T]$, поэтому $l(F)(x) \equiv 0$ в $\Omega$.

Пусть теперь $l(u)(x) \not \equiv 0$ в $\Omega$. Тогда по лемме 6 и следствию 5 имеем $u(x, \tau)>0$ в $\Omega$. По следствию $4 l(u)(x)>0$ в $\Omega$. Лемма доказана.

ЗАмечание 2. Условие $F(\cdot, t) \in C\left([0, T] ; L_{p}(\Omega)\right)$ в лемме 8 может быть заменено на требование непрерывности для п.в. $x \in \Omega$ функции $F(x, t)$ по переменной $t \in[0, T]$.

\section{§ 4. Вывод операторного уравнения. Фредгольмовость задачи}

4.1. Операторное уравнение. Пусть выполнены условия (A), (B), (C). Тогда, как уже отмечалось, обратная задача (2.1)-(2.3) эквивалентно сводится к задаче нахождения пары $\{u(x, t) ; f(x)\}$ из условий $(2.4)-(2.6)$, в которых 
$f(x) \equiv l(\widetilde{h}) \widetilde{f}(x), h(x, t) \equiv \widetilde{h}(x, t) / l(\widetilde{h})$, и поэтому $\widetilde{h}(x, t) \widetilde{f}(x) \equiv h(x, t) f(x)$; функция $\chi \in W_{2}^{2}(\Omega)$. В силу условий согласования (см. условие $\left.(\mathrm{C})\right) B \chi(x)=0$, $x \in \partial \Omega$, т.е. функции $h(x, t)$ и $\chi(x)$ удовлетворяют условию (D). Операторы $L(t)$ и $B$ такие же, как в $(2.1),(2.2)$.

Для получения эквивалентного операторного уравнения в эллиптическом операторе $L(t)$ удобно выделить обратимую стационарную часть, записав $L(t)$ в виде

$$
\begin{aligned}
L(t) u & \equiv \sum_{i, j=1}^{n} \frac{\partial}{\partial x_{i}}\left(a_{i j}(x) \frac{\partial u}{\partial x_{j}}\right)+\lambda_{0} u+\sum_{i=1}^{n} b_{i}(x, t) \frac{\partial u}{\partial x_{i}}+\left(d(x, t)-\lambda_{0}\right) u \\
& \equiv L_{0} u+(\vec{b}(x, t), \nabla u)+\widetilde{d}(x, t) u
\end{aligned}
$$

с числом $\lambda_{0}<0$. Тогда эллиптическая краевая задача

$$
-L_{0} v(x)=g(x), \quad x \in \Omega, \quad B v(x)=0, \quad x \in \partial \Omega,
$$

при любой функции $g \in L_{2}(\Omega)$ имеет и притом единственное решение $v \in$ $W_{2}^{2}(\Omega)$ (см. [25; гл. $\left.\left.3, \S 9\right]\right)$. При каждом значении $t \in[0, T]$ определим оператор $\mathrm{G}(t): L_{2}(\Omega) \rightarrow L_{2}(\Omega)$ по правилу

$$
\mathrm{G}(t) f:=\rho(\cdot, t) u_{t}(\cdot, t ; f)-(\vec{b}, \nabla u)-\widetilde{d}(\cdot, t) u,
$$

где $u=u(x, t ; f)$ - решение прямой задачи $(2.4),(2.5)$ с данным $f \in L_{2}(\Omega)$. Из лемм 2-4 и их следствий получим, что при каждом $t \in[0, T]$ оператор $\mathrm{G}(t) \in \mathscr{L}\left(L_{2}(\Omega)\right)$, а семейство $\mathrm{G}(t)$ удовлетворят условиям леммы 1.

Перейдем к выводу операторного уравнения. Для этого запишем уравнение (2.4) в виде

$$
\mathrm{G}(t) f-L_{0} u=h f .
$$

Предположим теперь, что пара $\{u(x, t) ; f(x)\}$ является решением обратной задачи (2.4)-(2.6). Тогда, в частности, выполняется соотношение (4.4), в котором правая и левая части суть непрерывные на отрезке $[0, T]$ в норме $L_{2}(\Omega)$ функции. Поэтому законно подействовать на (4.4) оператором переопределения $l$ и получить такое следствие:

$$
\mathscr{B} f-l\left(L_{0} u\right)=l(h) f
$$

где $\mathscr{B} f:=\int_{0}^{T} \mathrm{G}(t) f d \mu(t), l\left(L_{0} u\right)=L_{0}(l(u))=L_{0} \chi$. Здесь мы воспользовались замкнутостью в $L_{2}(\Omega)$ оператора $L_{0}$, определенного на функциях из $W_{2}^{2}(\Omega)$ с соответствующими краевыми условиями (см. [25; гл. $3, \S 8]$ ), а также условием переопределения (2.6). Учитывая, что $l(h)(x) \equiv 1$ в $\Omega$, запишем искомое операторное уравнение

$$
\mathscr{B} f-L_{0} \chi=f .
$$

\section{2. Фредгольмовость обратной задачи.}

Теорема 1. Пусть выполненъ условия (А), (В), (C). Тогда обратная задача (2.1)-(2.3) эквивалентна линейному операторному уравнению (4.6) с вполне непрерывным в $L_{2}(\Omega)$ оператором $\mathscr{B}$. 
ДокАзАТЕЛьство. Как показано в $\S 2$, задача $(2.1)-(2.3)$ эквивалентно сводится к задаче $(2.4)-(2.6)$, где $f(x) \equiv l(\widetilde{h}) \widetilde{f}(x)$, а функции $h(x, t) \equiv \widetilde{h}(x, t) / l(\widetilde{h})$ и $\chi(x) \equiv \widetilde{\chi}(x)-l\left(u^{0}\right)$ удовлетворяют условию (D). Покажем, что обратная задача (2.4)-(2.6) эквивалентна уравнению (4.6). Действительно, если пара $\{u(x, t) ; f(x)\}$ - решение обратной задачи (2.4)-(2.6), то, повторяя рассуждения по выводу (4.6), убеждаемся, что $f \in L_{2}(\Omega)$ - решение операторного уравнения (4.6). Обратно, пусть $f \in L_{2}(\Omega)$ - решение (4.6). По $f(x)$ найдется единственным образом $u=u(x, t ; f)$ - решение прямой задачи $(2.4),(2.5)$, которое обладает в силу лемм 2-4 необходимыми дифференциальными свойствами. Для этих функций $u$ и $f$ выполняется равенство $(4.5)$ в $L_{2}(\Omega)$, а следовательно, и равенство

$$
\mathscr{B} f-L_{0}(l(u))=f,
$$

где $l(u) \in W_{2}^{2}(\Omega)$ и $B l(u)(x)=0, x \in \partial \Omega$. Последнее соотношение легко выводится из того, что $u(\cdot, t) \in C\left([0, T] ; W_{2}^{2}(\Omega)\right)$, и поэтому при всех $t \in[0, T]$ имеем $B u(x, t)=0, x \in \partial \Omega$. Вычитая теперь (4.6) из (4.7) получим верные равенства

$$
-L_{0}[l(u)-\chi](x)=0, \quad x \in \Omega, \quad B[l(u)-\chi](x)=0, \quad x \in \partial \Omega .
$$

Из единственности решения эллиптической краевой задачи (4.8) следует, что $l(u)(x)=\chi(x), x \in \Omega$. Таким образом, пара $\{u(x, t) ; f(x)\}$ является решением обратной задачи (2.4)-(2.6). Здесь $f(x)$ - решение уравнения (4.6), а $u=u(x, t ; f)$ - решение прямой задачи (2.4), (2.5) с этим $f$. Эквивалентность обратной задачи операторному уравнению установлена. Как отмечалось, семейство операторов $\mathrm{G}(t)$, определенное в (4.3), удовлетворяет условиям леммы 1. Отсюда получим, что $\mathscr{B}=\int_{0}^{T} \mathrm{G}(t) d \mu(t)$ является вполне непрерывным в $L_{2}(\Omega)$ оператором. Теорема доказана.

\section{§5. Корректность обратной задачи и оценка спектрального радиуса оператора $\mathscr{B}$}

\section{1. Достаточные условия корректности обратной задачи.}

Tеорема 2. Пусть оператор $L(t)$ в уравнении (2.1) имеет вид (2.7), въполнены условия (A), (B), (C), (E), функция $\mu(t)$ является неубывающей на $[0, T], h(x, t) \geqslant 0$ в $Q$, где $h(x, t) \equiv \widetilde{h}(x, t) / l(\widetilde{h})$, и выполняется хотя бы одно из следующих условии:

1. $h_{t}(x, t) \geqslant 0, d(x, t) \leqslant 0, d_{t}(x, t) \geqslant 0$ в $Q$;

2. $d \mu(t)=\omega(t) d t$ с функиией $\omega \in W_{1}^{1}(0, T)$ такой, что справедливо неравенство $(\omega(t) \varrho(x, t))_{t}^{\prime}+d(x, t) \omega(t) \leqslant 0$ в $Q$

3. $d \mu(t)=\omega(t) d t$ с функиией $\omega \in \mathrm{BV}[0, T]$ такой, что для всех $x \in \Omega$ функиия $\pi(x, t) \equiv \omega(t) \varrho(x, t)+\int_{0}^{t} d(x, \tau) \omega(\tau) d \tau$ является невозрастающей по $t \in[0, T]$.

Тогда существует и притом единственное решение $\widetilde{u} \in W_{2}^{2,1}(Q), \widetilde{f} \in L_{2}(\Omega)$ задачи (2.1)-(2.3) и справедлива оценка устойчивости $\|\widetilde{f}\|_{2, \Omega}+\left\|\widetilde{u}-u^{0}\right\|_{2, Q}^{(2,1)} \leqslant$ 
$C\left\|L_{0}\left[\widetilde{\chi}-l\left(u^{0}\right)\right]\right\|_{2, \Omega}$. Указанное решение $\widetilde{u}(x, t)$ обладает следующими дополнительными дифберенциальными свойствами:

$$
\widetilde{u} \in C\left([0, T] ; W_{2}^{2}(\Omega)\right), \quad \widetilde{u}_{t} \in C\left([0, T] ; L_{2}(\Omega)\right), \quad \widetilde{u}_{t} \in W_{2}^{2,1}\left(Q_{\varepsilon}\right),
$$

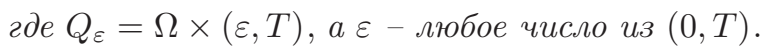

ДокАзАтЕльство. В условиях теоремы обратная задача (2.1)-(2.3) эквивалентно сводится к задаче (2.4)-(2.6) (см. §2), поэтому будем доказывать однозначную разрешимость именно этой задачи. В начале изложим схему доказательства утверждений теоремы 2, а потом уже проведем соответствующие рассуждения в каждом из случаев. Введем в рассмотрение оператор $\mathscr{B}: L_{2}(\Omega) \rightarrow L_{2}(\Omega)$ по правилу

$$
\mathscr{B} f:=l\left(\rho u_{t}-d \cdot u\right),
$$

где $u=u(x, t ; f)$ - решение $(2.4),(2.5)$ с данным $f \in L_{2}(\Omega)=: E$. По теореме 1 обратная задача (2.4)-(2.6) эквивалентна операторному уравнению в $E$

$$
\mathscr{B} f-L_{0} \chi=f .
$$

Отметим, что по условию нуль принадлежит резольвентному множеству $\rho\left(L_{0}\right)$ оператора $L_{0}$, определенного в (2.7). В теореме 1 установлено, что $\mathscr{B}$ - вполне непрерывный в пространстве $E$ оператор. В условиях 1,2 (или 3 ) теоремы 2 относительно $\mathscr{B}$ устанавливаются следующие свойства:

1. оператор $\mathscr{B} \geqslant 0$, т.е. $\mathscr{B} E_{+} \subseteq E_{+}$, где $E_{+}:=\left\{f \in L_{2}(\Omega) \mid f(x) \geqslant 0\right.$ в $\left.\Omega\right\}$;

2. спектральный радиус оператора $\mathscr{B}$ меньше единицы, т.е. $r(\mathscr{B})<1$;

3. в силу п. 2 существует $(I-\mathscr{B})^{-1} \in \mathscr{L}(E)$, и, следовательно, операторное уравнение (5.2) однозначно разрешимо.

Докажем утверждение теоремы 2 при выполнении одного из условий 2 или 3 , технически чуть более сложное. Для этого преобразуем выражение для $\mathscr{B} f=$ $l\left(\rho u_{t}-d \cdot u\right)$, учитывая, что $d \mu(t)=\omega(t) d t$ с функцией $\omega \in \operatorname{BV}[0, T]:$

$$
\begin{gathered}
\mathscr{B} f=\int_{0}^{T} \rho u_{t} d \mu(t)-\int_{0}^{T} d \cdot u d \mu(t)=I_{1}-I_{2}, \\
I_{1}:=\int_{0}^{T} \rho \omega u_{t} d t=\left.\rho \omega u\right|_{0} ^{T}-\int_{0}^{T} u d_{t}(\omega \rho), \\
I_{2}:=\int_{0}^{T} u d \cdot \omega d t=\int_{0}^{T} u d_{t}\left(\int_{0}^{t} d(x, \tau) \omega(\tau) d \tau\right),
\end{gathered}
$$

получим

$$
\mathscr{B} f=(\rho \omega u)(x, T)-\int_{0}^{T} u d_{t}\left(\omega \rho+\int_{0}^{t} d(x, \tau) \omega(\tau) d \tau\right) .
$$

Покажем, что $\mathscr{B} \geqslant 0$. Для этого возьмем произвольный элемент $f \geqslant 0$ из $L_{2}(\Omega)$ и решим задачу $(2.4),(2.5)$ с данным $f$. По лемме $5 u=u(x, t ; f) \geqslant 0$ в $Q$, а по условию 2 (3) теоремы 2 для любого $x \in \Omega$ функция ограниченной вариации $\pi(x, t)=\omega(t) \rho(x, t)+\int_{0}^{t} d(x, \tau) \omega(\tau) d \tau$ является невозрастающей по $t \in[0, T]$. 
Тогда из определения интеграла Римана-Стилтьеса имеем $\mathscr{B} f \geqslant 0$, т.е. $\mathscr{B} \geqslant 0$. Докажем, что $r(\mathscr{B})<1$. Предположим, что это не так, т.е. $r(\mathscr{B}) \geqslant 1$. Тогда по теореме Крейна-Рутмана (см. [28]) число $r(\mathscr{B})$ является собственным значением $\mathscr{B}$ с собственным вектором $f_{0} \geqslant 0$, т.е. $\mathscr{B} f_{0}=r(\mathscr{B}) f_{0}$. Заметим, что конус $E_{+}$всех неотрицательных функций из $L_{2}(\Omega)$ является замкнутым, воспроизводящим (см., например, [29]) и удовлетворяет условиям теоремы Крейна-Рутмана. Рассмотрим функцию $u=u\left(x, t ; f_{0}\right)$ - решение $(2.4),(2.5)$ с этим $f_{0}$. Повторяя вывод операторного уравнения (5.2), как и при доказательстве теоремы 1 , получим в $L_{2}(\Omega)$ равенство $\mathscr{B} f_{0}-L_{0} l(u)=f_{0}$. Учитывая, что $\mathscr{B} f_{0}=r(\mathscr{B}) f_{0}$, имеем

$$
-L_{0} l(u)=(1-r(\mathscr{B})) f_{0} \leqslant 0 .
$$

Оператор $L_{0}$ удовлетворяет условиям слабого принципа максимума для эллиптических уравнений (см. [30], [31]), из которого (с учетом $B l(u)=0$ на $\partial \Omega$ ) получаем $l(u) \leqslant 0$ в $\Omega$. Но $u=u\left(x, t ; f_{0}\right) \geqslant 0$, а функция $\mu(t)$ является неубывающей, поэтому $l(u) \geqslant 0$ в $\Omega$. Отсюда $l(u) \equiv 0$ в $\Omega$, а тогда по лемме 8 выполняется равенство

$$
l\left(h f_{0}\right)=l(h) f_{0}=f_{0}=0 \quad \text { в } \Omega .
$$

Получим противоречие с $f_{0} \neq \theta$, поскольку $f_{0}$ - собственный вектор оператора $\mathscr{B}$ (здесь $\theta$ - нулевой элемент $E$ ). Таким образом, $r(\mathscr{B})<1$, и поэтому существует $(I-\mathscr{B})^{-1} \in \mathscr{L}(E)$. Операторное уравнение $(5.2)$ имеет единственное решение, а по теореме 1 и обратная задача имеет и притом единственное решение. Утверждение теоремы 2 при выполнении одного из условий 2 или 3 доказано.

Докажем теперь утверждение теоремы 2 при выполнении условия 1. Это доказательство отличается от предыдущего только в первом пункте схемы, т.е. при обосновании свойства $\mathscr{B} \geqslant 0$. Возьмем $f \in L_{2}(\Omega)$ и $u(x, t ; f)$ - решение $(2.4),(2.5)$ с этим $f$. В силу свойств дополнительной гладкости функция $v=u_{t}(x, t ; f)$ принадлежит $V_{2}^{1,0}(Q)$ и удовлетворяет задаче (см. лемму 2)

$$
\begin{gathered}
\rho v_{t}-L_{0} v-d v+\rho_{t} v=d_{t} u(x, t)+h_{t} f(x), \quad(x, t) \in Q, \\
v(x, 0)=\frac{h(x, 0) f(x)}{\rho(x, 0)}, \quad x \in \Omega, \quad B v(x, t)=0, \quad(x, t) \in S .
\end{gathered}
$$

Обозначим $F(x, t):=d_{t}(x, t) u(x, t)+h_{t}(x, t) f(x)$, и пусть $f(x) \geqslant 0$ в $\Omega$. Тогда по лемме $5 u(x, t ; f) \geqslant 0$. По условию $d_{t}(x, t) \geqslant 0$ и $h_{t}(x, t) \geqslant 0$ в $Q$, а, кроме того, $v_{0}(x):=h(x, 0) f(x) / \rho(x, 0) \geqslant 0$ в $\Omega$. Отсюда по лемме 5 получим, что $u_{t}=v(x, t ; f) \geqslant 0$ в $Q$, и в силу того, что функция $\mu(t)$ является неубывающей на $[0, T]$, а $d(x, t) \leqslant 0$ в $Q$, имеем $\mathscr{B} f=l\left(\rho u_{t}-d \cdot u\right) \geqslant 0$ для всех $f \in E_{+}$, т.е. $\mathscr{B} \geqslant 0$. Далее без изменений повторяем рассуждения предыдущего пункта со слов "докажем, что $r(\mathscr{B})<1$ ”.

Поясним оценку устойчивости из теоремы 2. При выполнении условий теоремы $(I-\mathscr{B})^{-1} \in \mathscr{L}(E)$ существует единственное решение операторного уравнения $(5.5) f=(I-\mathscr{B})^{-1}\left(-L_{0} \chi\right)$, поэтому для решения справедлива оценка

$$
\|f\| \leqslant\left\|(I-\mathscr{B})^{-1}\right\| \cdot\left\|L_{0} \chi\right\|=C\left\|L_{0} \chi\right\| .
$$


В силу оценки устойчивости прямой задачи $(2.4),(2.5)$ в $W_{2}^{2,1}(Q)$ имеем неравенство $\|u\|_{2, Q}^{(2,1)} \leqslant C_{1}\|f\|$, здесь $\|f\|-$ норма в $L_{2}(\Omega)$. Отсюда выводим оценку теоремы 2. Доказательство дополнительных дифференциальных свойств решения $u(x, t)$ дано в леммах $2-4$. Теорема полностью доказана.

ЗАмечание 3 . Требование $|l(\widetilde{h})(x)| \geqslant \delta>0$ в $\Omega$, фигурирующее в условии $(\mathrm{C})$, существенно для корректности задачи (2.1)-(2.3). Его нарушение приводит к потере свойства устойчивости решения (подробнее см. [32]). Однако для единственности решения это требование может быть ослаблено.

5.2. Следствие из теоремы 2. В процессе доказательства теоремы 2 фактически установлен следующий результат, который может быть полезен для численного решения задачи, поскольку дает сходящийся итерационный процесс построения искомой функции $f(x)$.

СлеДСтвиЕ 6. В условиях теоремы 2 спектралъный радиус оператора $\mathscr{B}$ из (5.1) менъше единицы. Решение операторного уравнения (5.2) может быть получено в виде ряда Неймана $f=\sum_{k=0}^{\infty} \mathscr{B}^{k}\left(-L_{0} \chi\right)$, а функция $u=u(x, t ; f)$ как решение прямой задачи (2.4), (2.5) с этим $f$.

ДокАЗАТЕЛьство. Действительно, неравенство $r(\mathscr{B})<1$ установлено при доказательстве теоремы 2, а поэтому (см., например, справочник [29; гл. 5, §3]) ряд Неймана $f=(I-\mathscr{B})^{-1}\left(-L_{0} \chi\right)=\sum_{k=0}^{\infty} \mathscr{B}^{k}\left(-L_{0} \chi\right)$ сходится и дает искомое решение операторного уравнения $f \in L_{2}(\Omega)$. Получаем пару функций $f(x)$ и $u(x, t ; f)$, которая по теореме 1 является решением обратной задачи $(2.4)-(2.6)$. Оператор $\mathscr{B}$ задан в (5.1) и выражается через решение прямой задачи, поэтому для вычисления $f$ удобно ввести последовательность $f_{k}=f_{0}+\mathscr{B} f_{k-1}$ с начальным приближением $f_{0}=-L_{0} \chi$, которая будет сходиться к искомому решению в норме $L_{2}(\Omega)$. Следствие 6 доказано.

ЗАмЕчАНИЕ 4. Изложенный метод доказательства корректности обратной задачи, основанный на оценке спектрального радиуса, впервые применялся в работе [9] для исследования методами теории полугрупп задачи с финальным и интегральным наблюдением. Здесь этот метод перенесен на более общее условие нелокального наблюдения и на нестационарные параболические уравнения. Отметим еще, что для обратной задачи с финальным наблюдением метод, основанный на оценке спектрального радиуса, применялся в [33]. В [34] методом позитивности доказана теорема о корректности обратной задачи с финальным наблюдением. В качестве следствия из нее была также получена оценка спектрального радиуса.

\section{§ 6. Достаточные условия единственности решения}

6.1. Условие неизменности знака по переменной $t$. Для описания результатов по единственности решения обратной задачи нам понадобится следующее

ОПредЕлЕниЕ 3 . Пусть функция $\widetilde{h}(x, t)$ для всех $x \in \Omega$ непрерывна по переменной $t \in[0, T]$. Будем говорить, что функция $\widetilde{h}(x, t)$ не изменяет знак по $t \in[0, T]$, если для любой точки $x_{0} \in \Omega$ возможен только один из трех случаев: 
1. либо $\widetilde{h}\left(x_{0}, t\right) \equiv 0$ на $[0, T]$;

2. либо $\widetilde{h}\left(x_{0}, t\right) \geqslant 0$ на $[0, T]$ и $\widetilde{h}\left(x_{0}, t\right) \not \equiv 0$;

3. либо $\widetilde{h}\left(x_{0}, t\right) \leqslant 0$ на $[0, T]$ и $\widetilde{h}\left(x_{0}, t\right) \not \equiv 0$.

Все точки $x_{0} \in \Omega$, для которых имеет место случай 1 , образуют множество $\Omega_{0}$, случай 2 - множество $\Omega_{+}$, а случай 3 - множество $\Omega_{-}$. При этом $\Omega=\Omega_{0} \cup$ $\Omega_{+} \cup \Omega_{-}$и какие-то (не более двух) из множеств могут быть пустыми. Отметим, что в этом случае удобно ввести такую функцию $\varphi_{0}(x)$ ("функцию знака" для $\widetilde{h}(x, t))$, что

$$
\varphi_{0}(x)=\left\{\begin{array}{lll}
1, & \text { если } & x \in \Omega_{0} \cup \Omega_{+}, \\
-1, & \text { если } & x \in \Omega_{-} .
\end{array}\right.
$$

ЗАмЕчание 5 . По построению $\varphi_{0}^{2}(x) \equiv 1$ в $\Omega$. Кроме того, если $\mu(t)$ является неубывающей на $[0, T], \bigvee_{0}^{T}(\mu)>0$ и в области $\Omega$ выполнено неравенство $|l(\widetilde{h})(x)|>0$, то $\varphi_{0}(x) \equiv \operatorname{sgn} l(\widetilde{h})(x)$ в $\Omega$ (см. лемму 9 ниже). В общем случае при фиксированном $t_{0} \in[0, T] \operatorname{sgn} \widetilde{h}\left(x, t_{0}\right)$ может не совпадать с функцией знака $\varphi_{0}(x)$. В качестве примера возьмем функцию $\widetilde{h}(x, t)$ следующего вида:

$$
\widetilde{h}(x, t)= \begin{cases}0, & \text { если }(x, t) \in \bar{Q}_{\tau}=\left[-\frac{\pi}{4}, \frac{\pi}{4}\right] \times[0, \tau], \\ \eta(t) \sin (x(t-\tau)), & \text { если }(x, t) \in\left[-\frac{\pi}{4}, \frac{\pi}{4}\right] \times[\tau, \tau+2] .\end{cases}
$$

Здесь $\eta(t) \not \equiv 0$ - гладкая функция такая, что $0 \leqslant \eta(t) \leqslant 1$, причем $\eta(t) \equiv 0$ на $[0, \tau], \tau>0$. Для такой функции $\widetilde{h}(x, t)$ при фиксированном $t_{0} \in[0, T]$ $\operatorname{sgn} \widetilde{h}\left(x, t_{0}\right)$ зависит от $t_{0}$, но она не изменяет знак по переменной $t$ в смысле определения 3 и ее "функция знака" $\varphi_{0}(x)$ имеет вид

$$
\varphi_{0}(x)= \begin{cases}1, & \text { если } x \in\left[0, \frac{\pi}{4}\right] \\ -1, & \text { если } x \in\left[-\frac{\pi}{4}, 0\right) .\end{cases}
$$

ЗАмечАниЕ 6 . Если $\widetilde{h}(x, t)$ не изменяет знак по переменной $t \in[0, T]$, а $\varphi_{0}(x)$ - ее функция знака, то для $h(x, t):=\widetilde{h}(x, t) \varphi_{0}(x)$ всюду в $Q$ выполняется неравенство $h(x, t) \geqslant 0$. Это следует из построения функции $\varphi_{0}(x)$ в определении 3.

Лемма 9. Пусть $\widetilde{h}(x, t)$ не изменяет знак по переменной $t \in[0, T], \mu(t)$ удовлетворяет условиям (3.15) и справедливо неравенство $|l(\widetilde{h})(x)|>0$ в $\Omega$. Тогда $\varphi_{0}(x) \equiv \operatorname{sgn} l(\widetilde{h})(x)$, a $l(h)(x)>0$ в $\Omega$, где $\varphi_{0}(x)-$ функиия знака $\widetilde{h}(x, t)$.

ДокАЗАТЕЛЬство. Так как $|l(\widetilde{h})(x)|>0$ п.в. в $\Omega$, то множество $\Omega_{0}$ из определения 3 является пустым или $\operatorname{mes}_{n} \Omega_{0}=0$, поэтому для п.в. точек $x_{0} \in \Omega$ возможны лишь два случая: $l(\widetilde{h})\left(x_{0}\right)>0$ или $l(\widetilde{h})\left(x_{0}\right)<0$. В первом случае $\widetilde{h}\left(x_{0}, t\right) \geqslant 0$ и $\widetilde{h}\left(x_{0}, t\right) \not \equiv 0$ на $[0, T]$, т.е. $x_{0} \in \Omega_{+}, \varphi_{0}\left(x_{0}\right)=1$. Во втором случае $\widetilde{h}\left(x_{0}, t\right) \leqslant 0$ и $\widetilde{h}\left(x_{0}, t\right) \not \equiv 0$ на $[0, T]$, т.е. $x_{0} \in \Omega_{-}, \varphi_{0}\left(x_{0}\right)=-1$. Отсюда $l(h)(x) \equiv \varphi_{0}(x) l(\widetilde{h})(x) \equiv|l(\widetilde{h})(x)|>0$ в $\Omega$. Лемма доказана. 
Если $\widetilde{h}(x, t)$ изменяет знак по переменной $t \in[0, T]$, то единственности решения обратной задачи (2.1)-(2.3) в общем случае нет. Покажем, что для функции $\widetilde{h}(x, t)$, не изменяющей знак по $t \in[0, T]$, обратная задача $(2.1)-(2.3)$ сводится к случаю $h(x, t) \geqslant 0$. Ограничимся при этом однородной обратной задачей.

Лемма 10. Пусть выполненъ условия (A), (B), функиия $\widetilde{h}(x, t)$ не изменяет знак по переменной $t \in[0, T], a \mu(t)$ удовлетворяет условиям (3.15). Тогда однородная обратная задача $(2.1)-(2.3)$ с функциями $\widetilde{g}=\widetilde{u}_{0}=\widetilde{\beta}=\widetilde{\chi}=0$ эквивалентна обратной задаче нахождения $\{u(x, t) ; f(x)\}$ из условий

$$
\begin{gathered}
\rho(x, t) u_{t}(x, t)-L(t) u(x, t)=h(x, t) f(x), \quad(x, t) \in Q, \\
u(x, 0)=0, \quad x \in \Omega, \quad B u(x, t)=0, \quad(x, t) \in S, \\
l(u)=0, \quad x \in \Omega,
\end{gathered}
$$

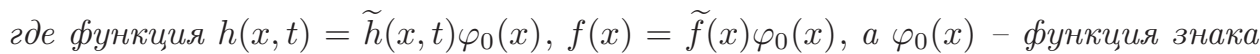
$\widetilde{h}(x, t)$, причем $h, h_{t} \in L_{\infty, 2}(Q)$, a $h(x, t) \geqslant 0$ в $Q$.

ДокАЗАТЕЛЬСТво. Поскольку $\varphi_{0}^{2}(x) \equiv 1$ в $\Omega$, то справедливо тождество $\widetilde{h}(x, t) \tilde{f}(x) \equiv h(x, t) f(x)$, по замечанию 6 имеем $h(x, t) \geqslant 0$ в $Q$. Предположив, что пара функций $\{\widetilde{u}(x, t) ; \widetilde{f}(x)\}$ - решение однородной обратной задачи, получим, что пара $\{u(x, t) ; f(x)\}$ - это решение $(6.1)-(6.3)$ и обратно, где $f(x) \equiv \widetilde{f}(x) \varphi_{0}(x)$, а $u(x, t) \equiv \widetilde{u}(x, t)$. Таким образом, между множествами решений указанных обратных задач установлено взаимно однозначное соответствие. Лемма доказана.

6.2. Теорема единственности решения задачи (2.1)-(2.3). Единственность решения линейной обратной задачи (2.1)-(2.3) стандартным способом сводится к отсутствию ненулевых решений этой задачи с функциями $\widetilde{g}=\widetilde{u}_{0}=$ $\widetilde{\beta}=\widetilde{\chi}=0$. При условии, что $\widetilde{h}(x, t)$ не изменяет знак по переменной $t \in[0, T]$, такая однородная обратная задача по лемме 10 эквивалентна задаче (6.1)-(6.3) с функцией $h(x, t) \geqslant 0$ в $Q$, удовлетворяющей условиям $h, h_{t} \in L_{\infty, 2}(Q)$. В связи с этим результаты, относящиеся к однородной обратной задаче, будем формулировать для преобразованной задачи (6.1)-(6.3).

Tеорема 3. Пусть оператор $L(t)$ в уравнении (6.1) имеет вид (2.7), выполнены условия (A), (E), $h, h_{t} \in L_{\infty, 2}(Q)$, функиия $\mu(t)$ удовлетворяет (3.15), $h(x, t) \geqslant 0$ в $Q$, a $l(h)(x)>0$ в $\Omega$. Предположим дополнительно, что выполняется хотя бы одно из условии:

1. $h_{t}(x, t) \geqslant 0, d(x, t) \leqslant 0, d_{t}(x, t) \geqslant 0$ в $Q$;

2. $d \mu(t)=\omega(t) d t$ с функцией $\omega \in \mathrm{BV}[0, T]$ такой, что для всех $x \in \Omega$ функиия $\pi(x, t) \equiv \omega(t) \varrho(x, t)+\int_{0}^{t} d(x, \tau) \omega(\tau) d \tau$ является невозрастающей по переменной $t \in[0, T]$.

Тогда однородная обратная задача (6.1)-(6.3) имеет лишь нулевое решение $u=0 u f=0$.

ДокАзАтельство. Пусть пара $\{u(x, t) ; f(x)\}$ - это какое-то решение обратной задачи (6.1)-(6.3). Рассмотрим, следуя работе [3], функции $f^{+}(x):=$ 
$\max \{0, f(x)\}$ и $f^{-}(x):=\max \{0,-f(x)\}$, а также функции $u^{ \pm}(x, t)$ как решения соответствующих задач

$$
\begin{gathered}
\rho(x, t) u_{t}^{ \pm}(x, t)-L_{0} u^{ \pm}(x, t)-d(x, t) u^{ \pm}(x, t)=h(x, t) f^{ \pm}(x), \quad(x, t) \in Q, \\
u^{ \pm}(x, 0)=0, \quad x \in \Omega, \quad B u^{ \pm}(x, t)=0, \quad(x, t) \in S .
\end{gathered}
$$

Из результатов [21; гл. $4, \S 9$ и гл. $3, \S 6]$ следует, что $u^{ \pm} \in W_{2}^{2,1}(Q)$, причем из слабого принципа максимума для параболических уравнений имеем $u^{ \pm}(x, t) \geqslant 0$ в $Q$ (см. [24; гл. $\left.\left.4, \S \S 7,10\right]\right)$. Так как $f(x)=f^{+}(x)-f^{-}(x)$, то из единственности решения прямой задачи $(6.1),(6.2) u(x, t)=u^{+}(x, t)-u^{-}(x, t)$, откуда (с учетом $(6.3)) l\left(u^{+}\right)(x)=l\left(u^{-}\right)(x) \equiv \bar{\chi}(x) \geqslant 0$ в $\Omega$, где $\bar{\chi} \in W_{2}^{2}(\Omega)$ и $B \bar{\chi}(x)=0$ на $\partial \Omega$. Дальнейшие рассуждения различны для условий 1 и 2 теоремы 3, поэтому проведем их для каждого из этих случаев отдельно.

Случай 1. Дадим доказательство утверждения теоремы 3 при выполнении условия 2. В силу лемм 2-4 функции $u^{ \pm}(x, t)$ обладают дополнительными дифференциальными свойствами, поэтому законно подействовать оператором переопределения $l$ на правую и левую части уравнения (6.4), при этом получим верные равенства в $L_{2}(\Omega)$

$$
l\left(\rho u_{t}^{ \pm}\right)-L_{0} l\left(u^{ \pm}\right)-l\left(d \cdot u^{ \pm}\right)=l(h) f^{ \pm} .
$$

Здесь мы воспользовались замкнутостью оператора $L_{0}$. Сгруппируем слагаемые и запишем эти равенства в следующем виде:

$$
-L_{0} \bar{\chi}=l(h) f^{ \pm}-l\left(\rho u_{t}^{ \pm}-d \cdot u^{ \pm}\right) .
$$

Правая часть равенства (6.6) одинакова для функций $f^{+}(x)$ и $f^{-}(x)$, обозначим ее $\varphi(x):=l(h) f^{ \pm}-l\left(\rho u_{t}^{ \pm}-d \cdot u^{ \pm}\right)$и проанализируем подробнее. Второе слагаемое в (6.6) преобразуем к виду (см. доказательство теоремы 2)

$$
l\left(\rho u_{t}^{ \pm}-d \cdot u^{ \pm}\right)=\left(\rho \omega u^{ \pm}\right)(x, T)-\int_{0}^{T} u^{ \pm} d_{t}\left(\omega \rho+\int_{0}^{t} d(x, \tau) \omega(\tau) d \tau\right) .
$$

По лемме 5 функции $u^{ \pm}(x, t) \geqslant 0$, а по условию 2 для любого $x \in \Omega$ функция

$$
\pi(x, t)=\omega(t) \rho(x, t)+\int_{0}^{t} d(x, \tau) \omega(\tau) d \tau \text { является невозрастающей по } t \in[0, T] .
$$

Поэтому $l\left(\rho u_{t}^{ \pm}-d \cdot u^{ \pm}\right) \geqslant 0$ в $\Omega$. Таким образом, функция $\varphi(x)$ удовлетворяет неравенствам $\varphi(x) \leqslant l(h) f^{ \pm}(x)$. Тогда $\varphi(x) \leqslant \min \left\{l(h) f^{+}(x), l(h) f^{-}(x)\right\} \equiv 0$ в $\Omega$. Отсюда, учитывая $(6.6)$, для функции $\bar{\chi}(x)$ получим систему

$$
-L_{0} \bar{\chi}(x) \leqslant 0 \quad \text { в } \Omega, \quad B \bar{\chi}(x)=0 \quad \text { на } \partial \Omega .
$$

Из слабого принципа максимума для эллиптического оператора $L_{0}$ (см. [30], [31]) следует, что $\bar{\chi}(x) \leqslant 0$ в $\Omega$, но $\bar{\chi}(x)=l\left(u^{ \pm}\right)(x) \geqslant 0$, поэтому $\bar{\chi}(x) \equiv 0$ в $\Omega$. По лемме $8 l\left(F^{ \pm}\right):=l\left(h f^{ \pm}\right) \equiv 0$, т.е. $l(h) f^{ \pm}(x) \equiv 0$, а по условию теоремы 3 $l(h)>0$ в $\Omega$. Следовательно, $f^{ \pm}(x) \equiv 0, f(x) \equiv 0$ в $\Omega$ и в силу единственности решения прямой задачи $(6.1),(6.2) u(x, t) \equiv 0$ в $Q$. Случай 1 полностью разобран. 
Случай 2. Проведем доказательство утверждения теоремы 3 при выполнении условия 1. Повторив рассуждения случая 1, выведем равенства (6.6), правую часть которых также обозначим $\varphi(x)$. Вначале докажем, что при выполнении условия 1 теоремы 3 справедливо неравенство

$$
l\left(\rho u_{t}^{ \pm}-d \cdot u^{ \pm}\right) \geqslant 0 \quad \text { в } \Omega .
$$

Действительно, в силу дополнительной гладкости функций $v^{ \pm}(x, t)=u_{t}^{ \pm}(x, t)$ они принадлежат $V_{2}^{1,0}(Q)$ и являются решениями соответствующих задач

$$
\begin{array}{ll}
\rho v_{t}^{ \pm}-L_{0} v^{ \pm}-d v^{ \pm}+\rho_{t} v^{ \pm}=d_{t} u^{ \pm}(x, t)+h_{t} f^{ \pm}(x), \quad(x, t) \in Q, \\
v^{ \pm}(x, 0)=\frac{h(x, 0) f^{ \pm}(x)}{\rho(x, 0)}, \quad x \in \Omega, \quad B v^{ \pm}(x, t)=0, \quad(x, t) \in S .
\end{array}
$$

По условию теоремы, так же как при доказательстве в случае $1, u^{ \pm}(x, t) \geqslant 0$ в $Q, d_{t}(x, t) \geqslant 0, d(x, t) \leqslant 0, h_{t}(x, t) \geqslant 0$ в $Q$, а $h(x, 0) f^{ \pm}(x) / \rho(x, 0) \geqslant 0$ в $\Omega$. Тогда по лемме 5 получим, что $u_{t}^{ \pm}(x, t)=v^{ \pm}(x, t) \geqslant 0$ в $Q$. Функция $\mu(t)$ является неубывающей, отсюда вытекает справедливость неравенства (6.7). Далее необходимо дословно повторить рассуждения случая 1 со слов "поэтому $l\left(\rho u_{t}^{ \pm}-d \cdot u^{ \pm}\right) \geqslant 0$ в $\Omega$ ". Случай 2 разобран, и теорема 3 полностью доказана.

ЗАмЕчАниЕ 7 . В условиях теоремы 3 функция $l(h)(x)$ может обращаться в нуль на множестве $\Omega_{0} \subset \mathbb{R}^{n}$ (см. определение 3 ), но его мера Лебега равна нулю. Если этого не требовать, т.е. допустить, что $\operatorname{mes}_{n} \Omega_{0}>0$, то свойство единственности, вообще говоря, нарушается. Если же остальные условия теоремы 3 сохраняются, то удается описать ядро обратной задачи, т.е. множество пар $\{u(x, t) ; f(x)\}$, являющихся решениями однородной обратной задачи. Подробнее об этом см. $\S 7$.

ЗАмечАниЕ 8. Схема рассуждения в доказательстве теоремы 3 применялась ранее для стационарного оператора или для частных случаев оператора наблюдения $l(u)$ (см. [3], [8]-[10], [14], [34]), получила название метода позитивности.

6.3. Теорема единственности для второй краевой задачи. Условие $L_{0} 1 \not \equiv 0$ в $\Omega$, присутствующее в теореме 3 в случае второй краевой задачи, может быть снято. Это требует изменений в рассуждениях теоремы 3 , поэтому данный случай выделен в отдельное утверждение.

Tеорема 4. Пусть оператор $L(t)$ в уравнении (6.1) имеет вид (2.7) где $c(x) \equiv 0$ в $\Omega$, оператор краевых условий $B u=\frac{\partial}{\partial N} u$, выполнены условия (A), $h, h_{t} \in L_{\infty, 2}(Q)$, бункиия $\mu(t)$ удовлетворяет (3.15) и справедливы неравенства $h(x, t) \geqslant 0$ в $Q, l(h)(x)>0$ в $\Omega$. Предположим дополнительно, что выполняется хотя бъ одно из условий:

1. $h_{t}(x, t) \geqslant 0, d(x, t) \leqslant 0, d_{t}(x, t) \geqslant 0$ в $Q$;

2. $d \mu(t)=\omega(t) d t$ с функиией $\omega \in \mathrm{BV}[0, T]$ такой, что для всех $x \in \Omega$ функция $\pi(x, t):=\omega(t) \varrho(x, t)+\int_{0}^{t} d(x, \xi) \omega(\xi) d \xi$ является невозрастающей по $t \in[0, T]$ и при этом для каждого $x \in \Omega$ число $\tau \in(0, T]$ является точкой роста слева функиии $\pi_{0}(x, t):=-\pi(x, t)$, здесь $\tau-$ крайняя правая точка роста $\mu(t)$; 
3. $d \mu(t)=\omega(t) d t$ с функиией $\omega \in \mathrm{BV}[0, T]$ такой, что для всех $x \in \Omega$ функиия $\pi(x, t)=\omega(t) \varrho(x, t)+\int_{0}^{t} d(x, \xi) \omega(\xi) d \xi$ является невозрастающей по $t \in[0, T]$, а коэббиииент $d(x, t) \leqslant 0$ в $Q$.

Тогда однородная обратная задача (6.1)-(6.3) имеет лишь нулевое решение $u=0, f=0$.

ДоказАтельство. Как в теореме 3, выведем соотношение (6.6) и для функции $\bar{\chi}(x):=l\left(u^{ \pm}\right)(x)$ получим систему

$$
-L_{0} \bar{\chi}(x) \leqslant 0 \quad \text { в } \Omega, \quad \frac{\partial}{\partial N} \bar{\chi}(x)=0 \quad \text { на } \partial \Omega .
$$

Тогда из слабого принципа максимума для эллиптического оператора $L_{0}$ (см. $[30])$ следует, что либо $\bar{\chi}(x) \leqslant 0$ в $\Omega$, либо $\bar{\chi}(x) \equiv$ const в $\Omega$. В первом случае, как и при доказательстве теоремы $3, \bar{\chi}(x) \equiv 0$ в $\Omega$ и далее $f(x) \equiv 0$ в $\Omega$. В силу же единственности решения прямой задачи (6.1), (6.2) $u(x, t) \equiv 0$ в $Q$.

Во втором случае $\bar{\chi}(x) \equiv$ const $\equiv \bar{\chi}$ в $\Omega$, причем по леммам 5 и $8 \bar{\chi}>0$. Дальнейшие рассуждения разделим на два пункта, проведя их отдельно при выполнении условий 1 и 2 (или 3 ) соответственно.

Начнем с условия 1 доказываемой теоремы. Предположим вначале, что $f^{-}(x) \equiv 0$ в $\Omega$. Тогда $u=u^{+}$и $l(u)=l\left(u^{+}\right)=\bar{\chi}>0$. Это противоречит условию $l(u)=0$ в $\Omega$. Аналогично рассматривается случай $f^{+}(x) \equiv 0$ в $\Omega$.

Предположим теперь, что $f^{+}(x) \not \equiv 0$ и $f^{-}(x) \not \equiv 0$ в $\Omega$. Логически возможны следующие два случая.

a) Пусть $l\left(\rho u_{t}^{-}\right) \equiv 0$ в $\Omega$. Отсюда следует, что $l\left(u_{t}^{-}\right) \equiv 0$ в $\Omega$, и по лемме 8 $v^{-}(x, t):=u_{t}^{-}(x, t) \equiv 0$ в $Q_{\tau}$, а кроме того, справедливы равенства

$$
\begin{cases}h(x, 0) f^{-}(x) \equiv 0 & \text { в области } \Omega, \\ h_{t}(x, t) f^{-}(x)+d_{t}(x, t) u^{-}(x, t) \equiv 0 & \text { в цилиндре } Q_{\tau} .\end{cases}
$$

Напомним, что функции $v^{ \pm}(x, t)=u_{t}^{ \pm}(x, t) \in V_{2}^{1,0}(Q)$ удовлетворяют задаче $(6.8),(6.9)$, а по условию 1 имеем $d(x, t) \leqslant 0, h_{t}(x, t) \geqslant 0, d_{t}(x, t) \geqslant 0$ в $Q$. Функция $\mu(t)$ является неубывающей, а из условий (6.10) следует, что $h_{t}(x, t) f^{-}(x) \equiv 0$ в $Q_{\tau}$. Отсюда $h(x, t) f^{-}(x)=\int_{0}^{t} h_{\xi}(x, \xi) d \xi f^{-}(x) \equiv 0$ в $Q_{\tau}$, и тогда $l(h) f^{-}(x) \equiv 0$, т.е. $f^{-}(x) \equiv 0$ в области $\Omega$, а этот случай уже рассмотрен.

b) Пусть теперь $l\left(\rho u_{t}^{-}\right) \not \equiv 0$ в $\Omega$. Тогда $l\left(u_{t}^{-}\right) \not \equiv 0$ в $\Omega$ и $v^{-}(x, t)=u_{t}^{-}(x, t) \not \equiv 0$ в $Q_{\tau}$, а по лемме 6 и следствию $4 l\left(u_{t}^{-}\right)>0$ в $\Omega$. Поэтому $l\left(\rho u_{t}^{-}\right)>0$ и $l(h) f^{-}(x)=l\left(\rho u_{t}^{-}-d u^{-}\right)>0$ в $\Omega$, т.е. $f^{+}=0, f=-f^{-}, u=-u^{-}, l(u)=-\bar{\chi}<0$, что противоречит условию $l(u)=0$ в $\Omega$.

Таким образом, теорема при выполнении условия 1 доказана.

Установим ее справедливость при выполнении условий 2 (или 3). Пусть $\bar{\chi}(x) \equiv$ const $>0$ в $\Omega, f^{+}(x) \not \equiv 0, f^{-}(x) \not \equiv 0$. Запишем равенство (6.6) в виде

$$
l(h) f^{ \pm}=\left(\rho \omega u^{ \pm}\right)(x, T)-\int_{0}^{T} u^{ \pm} d_{t}\left(\omega \rho+\int_{0}^{t} d(x, \xi) \omega(\xi) d \xi\right)
$$

где функция $\pi(x, t)=\omega(t) \varrho(x, t)+\int_{0}^{t} d(x, \xi) \omega(\xi) d \xi$ для всех $x \in \Omega$ является невозрастающей по $t \in[0, T]$. Функция $\omega(t) \in \mathrm{BV}[0, T]$ и $\omega(t) \geqslant 0$ по условию. 
Предположим вначале, что $\omega(T)>0$. Так как $h(x, t) f^{ \pm}(x) \not \equiv 0$ в $Q$, то по лемме 5 имеем $u^{ \pm}(x, T)>0$ в $\Omega$. Учитывая еще, что $\rho(x, T) \geqslant \rho_{0}>0$, из (6.11) получаем неравенства $l(h) f^{ \pm}(x)>0$ в $\Omega$, а это противоречит тождеству $f^{+}(x) f^{-}(x) \equiv 0$ в $\Omega$, справедливому по построению $f^{ \pm}(x)$.

По условию $\tau=\sup \left\{t_{*} \in(0, T] \mid t_{*}-\right.$ точка роста $\mu(t)$ слева $\}$. Как отмечалось выше (см. определение 2$)$, само число $\tau$ является точкой роста $\mu(t)$ слева. По условию 2 функция $\pi_{0}(x, t)=-\omega(t) \varrho(x, t)-\int_{0}^{t} d(x, \xi) \omega(\xi) d \xi$ для всех $x \in \Omega$ является неубывающей по переменной $t \in[0, T]$, а $\tau$ является ее точкой роста при всех $x \in \Omega$. Так как $h(x, t) f^{ \pm}(x) \not \equiv 0$ в $Q_{\tau}$, то по лемме 6 имеем $u^{ \pm}(x, \tau)>0$ в $\Omega$, а тогда по следствию 4 из (6.11) выводим справедливость неравенств

$$
l(h) f^{ \pm} \geqslant \int_{0}^{T} u^{ \pm}(x, t) d_{t} \pi_{0}(x, t)>0 \quad \text { в } \Omega .
$$

Это противоречит тождеству $f^{+}(x) f^{-}(x) \equiv 0$ в $\Omega$.

Для доказательства теоремы в случае 3 нам понадобится следующая

Лемма 11. Пусть $\mu(t)$ удовлетворяет $(3.15), \tau \in(0, T]-$ ее крайняя правая точка роста и выполнены неравенства $d(x, t) \leqslant 0$ в $Q, \omega(t) \geqslant 0$ на $[0, T]$. Тогда при всех $x \in \Omega$ число $\tau$ является точкой роста функиии $\pi_{0}(x, t)$ слева.

ДокАзАтельство. Пусть $\tau \in(0, T]$ - крайняя правая точка роста функции $\mu(t)$ слева. Если $d \mu(t)=\omega(t) d t$ с функцией $\omega \in \operatorname{BV}[0, T]$, то $\omega(t) \equiv 0$ на $[\tau, T]$ и для любого $\delta \in(0, \tau)$ имеем $\int_{\tau-\delta}^{\tau} \omega(t) d t=\bigvee_{\tau-\delta}^{\tau}(\mu)>0$ по определению точки роста $\tau$. Так как $\omega(t) \geqslant 0$, то это означает, что при каждом $\delta \in(0, \tau)$ функция $\omega(t) \not \equiv 0$ на $(\tau-\delta, \tau)$. Поэтому найдется бесконечно малая последовательность положительных чисел $\left\{\delta_{k}\right\}$ такая, что $\omega\left(\tau-\delta_{k}\right)>0$. Рассмотрим функцию $\pi_{0}(x, t)=-\omega(t) \varrho(x, t)-\int_{0}^{t} d(x, \tau) \omega(\tau) d \tau$. По условию она возрастает по переменной $t \in[0, T]$ для всех $x \in \Omega$. Отметим, что для всех $t \in[\tau, T] \pi_{0}(x, t)=\pi_{0}(x, \tau)=-\int_{0}^{\tau} d(x, \xi) \omega(\xi) d \xi$, т.е. $\pi_{0}(x, t)$ не имеет точек роста на $(\tau, T]$. Фиксируем произвольные $x \in \Omega$ и $\delta \in(0, \tau)$. Справедливы следующие соотношения:

$$
\begin{aligned}
\bigvee_{\tau-\delta}^{\tau}\left(\pi_{0}\right) & =-\int_{0}^{\tau} d(x, \xi) \omega(\xi) d \xi-\pi_{0}(x, \tau-\delta) \\
& =\omega(\tau-\delta) \rho(x, \tau-\delta)-\int_{\tau-\delta}^{\tau} d(x, \xi) \omega(\xi) d \xi \geqslant \omega(\tau-\delta) \rho_{0}
\end{aligned}
$$

Выберем теперь $\delta_{k}<\delta$ и для $x \in \Omega$ запишем неравенства

$$
\bigvee_{\tau-\delta}^{\tau}\left(\pi_{0}\right) \geqslant \bigvee_{\tau-\delta_{k}}^{\tau}\left(\pi_{0}\right) \geqslant \omega\left(\tau-\delta_{k}\right) \rho_{0}>0 .
$$

Таким образом, при каждом $x \in \Omega$ число $\tau$ является точкой роста функции $\pi_{0}(x, t)$ слева. Лемма 11 доказана.

В силу леммы 11 доказательство в случае 3 следует из случая 2. Теорема 4 полностью доказана. 
6.4. Разрешимость для второй краевой задачи как следствие теорем единственности и фредгольмовости. В теореме 1 доказано свойство фредгольмовости обратной задачи. В частности, из него следует, что единственность решения обратной задачи влечет существование решения в соответствующем классе. Для второй краевой задачи на этом пути получается результат, который не содержится в теореме 2 .

Теорема 5. Пусть оператор $L(t)$ в уравнении (2.4) имеет вид (2.7), где $c(x) \equiv 0$ в $\Omega, B u=\frac{\partial}{\partial N} u$, выполнены условия $(A),(D)$, функиия $\mu(t)$ удовлетворяет $(3.15), a h(x, t) \geqslant 0$ в $Q$. Предположим дополнительно, что выполняется хотя бы одно из условий:

1. $h_{t}(x, t) \geqslant 0, d(x, t) \leqslant 0, d_{t}(x, t) \geqslant 0$ в $Q$

2. $d \mu(t)=\omega(t) d t$ с функцией $\omega \in \mathrm{BV}[0, T]$ такой, что для всех $x \in \Omega$ функиия $\pi(x, t)=\omega(t) \varrho(x, t)+\int_{0}^{t} d(x, \xi) \omega(\xi) d \xi$ является невозрастающей по переменной $t \in[0, T]$ и при этом для каждого $x \in \Omega$ число $\tau \in(0, T]$ является точкой роста слева функиии $\pi_{0}(x, t)=-\pi(x, t)$, здесъ $\tau-$ крайняя правая точка роста $\mu(t)$;

3. $d \mu(t)=\omega(t) d t$ с функиией $\omega \in \mathrm{BV}[0, T]$ такой, что для всех $x \in \Omega$ функиия $\pi(x, t)=\omega(t) \varrho(x, t)+\int_{0}^{t} d(x, \xi) \omega(\xi) d \xi$ является невозрастающей по переменной $t \in[0, T]$, а коэффичиент $d(x, t) \leqslant 0$ в $Q$.

Тогда существует и притом единственное решение $u \in W_{2}^{2,1}(Q), f \in L_{2}(\Omega)$ обратной задачи (2.4)-(2.6), справедлива оценка устойчивости

$$
\|f\|_{2, \Omega}+\|u\|_{2, Q}^{(2,1)} \leqslant C\|\chi\|_{2, \Omega}^{(2)}
$$

Указанное решение и $(x, t)$ обладает следующими дополнительными дифференицальными свойствами:

$$
u \in C\left([0, T] ; W_{2}^{2}(\Omega)\right), \quad u_{t} \in C\left([0, T] ; L_{2}(\Omega)\right), \quad u_{t} \in W_{2}^{2,1}\left(Q_{\varepsilon}\right),
$$

где $Q_{\varepsilon}=\Omega \times(\varepsilon, T)$ при $\varepsilon \in(0, T)$.

ДоказАтельство. Выполнены все условия теорем 1 и 4. Обратная задача (2.4)-(2.6) эквивалентно сводится к операторному уравнению второго рода с вполне непрерывным оператором. Из единственности решения обратной задачи для краевых условий второго рода следует единственность решения этого операторного уравнения, откуда получаем его разрешимость, а вместе с ней и разрешимость обратной задачи. Оценка устойчивости и свойства дополнительной гладкости решения устанавливаются точно так же, как и в теореме 2. Теорема 5 доказана.

Для получения варианта теоремы 5 в терминах исходной задачи (2.1)-(2.3) необходимо дополнительно потребовать выполнения условий $(\mathrm{B}),(\mathrm{C})$ и проделать следующие замены $f(x)=l(\widetilde{h})(x) \widetilde{f}(x), u(x, t)=\widetilde{u}(x, t)-u^{0}(x, t), \chi(x)=$ $\widetilde{\chi}(x)-l\left(u^{0}\right)(x)$ и $h(x, t)=\widetilde{h}(x, t) l(\widetilde{h})^{-1}$. При этом функция $\widetilde{u}(x, t)$ будет обладать такой же дополнительной гладкостью, как и $u(x, t)$ в теореме 5. 


\section{$\S 7$. Описание ядра обратной задачи}

В этом параграфе, также как и в предыдущем, будем предполагать, что функция $\widetilde{h}(x, t)$ в исходной задаче $(2.1)-(2.3)$ не изменяет знак по $t \in[0, T]$ (см. определение 3), а тогда по лемме 10 однородная обратная задача эквивалентно сводится к задаче (6.1)-(6.3) с функцией $h(x, t) \geqslant 0$ в $Q$. В условиях доказанной выше теоремы 3 выполняется неравенство $l(h)>0$ для п.в. $x \in \Omega$ и $\operatorname{Ker} l(h)=\{\theta\}$, т.е. ядро оператора умножения на функцию $l(h)(x)$ в пространстве $L_{2}(\Omega)$ тривиально. Если в условиях теоремы 3 допустить, что $l(h)(x)=0$ на некотором множестве $\Omega_{0} \subseteq \Omega$ положительной меры, то $\operatorname{Ker} l(h)$ содержит ненулевые элементы и единственность решения обратной задачи нарушается, так как пара $u=0$ и $f \in \operatorname{Ker} l(h) \backslash\{\theta\}, f \in L_{2}(\Omega)$ является нетривиальным решением обратной задачи (6.1)-(6.3). Оказывается, что при выполнении остальных условий теоремы 3 такие пары исчерпывают все нетривиальные решения однородной обратной задачи.

ОПРЕДЕЛЕНИЕ 4. Множество пар $\{u(x, t) ; f(x)\}$, удовлетворяющих однородной обратной задаче (6.1)-(6.3) будем называть ядром обратной задачи и обозначать Ker. Если ядро содержит лишь пару $u=0, f=0$, то будем говорить, что оно тривиально, и это равносильно единственности решения линейной обратной задачи. В противном случае оно называется нетривиальным. Равенство $u=0$ в этом определении требует пояснения. Под этим равенством понимается $u(x, t) \equiv 0$ в $\bar{Q}_{\tau}$, где $\tau-$ крайняя правая точка роста функции $\mu(t)$.

Имеет место следующая теорема, в которой в отличие от теорем 3 и 4 отсутствует условие $l(h)(x)>0$ п.в. в $\Omega$.

Теорема 6. Пусть оператор $L(t)$ в уравнении (6.1) имеет вид (2.7), въполнень условия $(\mathrm{A}),(\mathrm{E}), h, h_{t} \in L_{\infty, 2}(Q)$, функиия $\mu(t)$ удовлетворяет $(3.15)$ и справедливо неравенство $h(x, t) \geqslant 0$ в $Q$. Предположим дополнительно, что выполняется хотя бы одно из условий:

1. $h_{t}(x, t) \geqslant 0, d(x, t) \leqslant 0, d_{t}(x, t) \geqslant 0$ в $Q$

2. $d \mu(t)=\omega(t) d t$ с функцией $\omega \in \mathrm{BV}[0, T]$ такой, что для всех $x \in \Omega$ функиия $\pi(x, t)=\omega(t) \varrho(x, t)+\int_{0}^{t} d(x, \xi) \omega(\xi) d \xi$ является невозрастающей по переменной $t \in[0, T]$.

Тогда имеют место следующие два утверждения.

1. Если пара $\{u ; f\} \in \mathbf{K e r}$, mo $f \in \operatorname{Ker} l(h), a u(x, t) \equiv 0$ в $\bar{Q}_{\tau}$.

2. Если $f \in \operatorname{Ker} l(h)$, mо пара $\{u ; f\} \in \operatorname{Ker}$, m.e. принадлежит ядру обратной задачи. Здесь $u=u(x, t ; f)$ - решение прямой задачи (6.1), (6.2) с данным $f$, причем $u(x, t) \equiv 0$ в $\bar{Q}_{\tau}$.

ДокАзАтельство. Более кратко утверждение теоремы 6 запишется так: пара $\{u ; f\} \in \operatorname{Ker}$ только тогда, когда $f \in \operatorname{Ker} l(h)$. Докажем необходимость. Предположим, что пара $\{u(x, t) ; f(x)\}$ является решением однородной обратной задачи (6.1)-(6.3). Как при доказательстве теоремы 3, введем функции $f^{ \pm}(x)$, $u^{ \pm}(x, t)$ и получим для них равенства

$$
l\left(\rho \cdot u_{t}^{ \pm}-d \cdot u^{ \pm}\right)-L_{0} l\left(u^{ \pm}\right)=l(h) f^{ \pm} .
$$


По условию $h(x, t) \geqslant 0$ в $Q, l(h)(x) \geqslant 0$ в $\Omega$. Обозначим $l\left(u^{ \pm}\right)(x) \equiv \bar{\chi}(x) \geqslant 0$ в $\Omega$ и, как при доказательстве теоремы 3 , в каждом из случаев 1 или 2 получим неравенства (6.7). Отсюда, повторяя рассуждения теоремы 3, в каждом из случаев 1 или 2 , найдем, что $l\left(u^{ \pm}\right)(x) \equiv 0$ и $l(h) f^{ \pm}(x) \equiv 0$ в $\Omega$, т.е. $f^{ \pm} \in \operatorname{Ker} l(h)$, а поэтому и $f=f^{+}-f^{-} \in \operatorname{Ker} l(h)$. Так как $l\left(u^{ \pm}\right)(x) \equiv 0$ в $\Omega$, то по лемме 8 $u^{ \pm}(x, t) \equiv 0$ и $u(x, t)=u^{+}(x, t)-u^{-}(x, t) \equiv 0$ в $\bar{Q}_{\tau}$. Здесь $\tau-$ крайняя правая точка роста функции $\mu(t)$. Необходимость доказана.

Докажем достаточность. Пусть $f \in \operatorname{Ker} l(h)$. Тогда $f^{ \pm} \in \operatorname{Ker} l(h)$, и из равенств (7.1) с учетом условий теоремы 6 получим, что

$$
-L_{0} l\left(u^{ \pm}\right)=-l\left(\rho u_{t}^{ \pm}-d \cdot u^{ \pm}\right) \leqslant 0 \quad \text { в } \Omega, \quad B l\left(u^{ \pm}\right)(x)=0 \quad \text { на } \partial \Omega .
$$

Как и выше, в силу слабого принципа максимума для эллиптических уравнений, имеем $l\left(u^{ \pm}\right)(x) \leqslant 0$ в $\Omega$, а по построению $u^{ \pm}(x, t) \geqslant 0$ в $\bar{Q}$. Следовательно, $l\left(u^{ \pm}\right)(x) \equiv 0$ в $\Omega$ и $u^{ \pm}(x, t) \equiv 0$ в $\bar{Q}_{\tau}$. Отсюда $u(x, t)=u^{+}(x, t)-u^{-}(x, t) \equiv 0$ в $\bar{Q}_{\tau}$ и $l(u)(x) \equiv 0$ в $\Omega$, т.е. $\{u ; f\} \in \mathbf{K e r}$. Теорема 6 доказана.

ЗАмЕчАниЕ 9. Если функция $\omega(t)$ в условии 1 принадлежит $W_{1}^{1}(0, T)$ и выполняется неравенство $(\omega \rho)_{t}^{\prime}+d \cdot \omega \leqslant 0$ в $Q$, то утверждение теоремы 6 очевидно сохранится. Отметим еще, что если $\tau=T$, т.е. $\bigvee_{T-\delta}^{T}(\mu)>0$ при любом $\delta \in(0, T)$, то $u(x, t) \equiv 0$ в $Q$. Это условие означает, что $\mu(t) \not \equiv$ const на $(T-\delta, T]$ при любом малом $\delta>0$, или в случае $d \mu(t)=\omega(t) d t$, что функция $\omega(t) \not \equiv 0$ на $(T-\delta, T]$. Другой случай, когда можно утверждать, что $u(x, t) \equiv 0$ в $Q$, описан ниже в следствии 7.

ЗАМЕчАНИЕ 10. В условиях теоремы 6 ядро обратной задачи полностью определяется ядром оператора умножения на функцию $l(h)(x)$, которое совпадает с $\operatorname{Ker} l(\widetilde{h})$. Теорема гарантирует лишь, что $u(x, t) \equiv 0$ в $\bar{Q}_{\tau}$. Примеры показывают, что вне $\bar{Q}_{\tau}$ функция $u(x, t)$ может не обращаться в тождественный нуль.

ПримеР 1. Возьмем функцию

$$
\mu(t)= \begin{cases}1, & \text { если } t \in\left[0, t_{1}\right), \\ 2, & \text { если } t \in\left[t_{1}, T\right] .\end{cases}
$$

Так как $t_{1} \in(0, T)$ - единственная точка роста $\mu(t)$, то $\tau=t_{1}$. Функцию $h(x, t)$ выберем по правилу

$$
h(x, t)= \begin{cases}0, & \text { если }(x, t) \in \bar{Q}_{\tau}, \\ \eta(t), & \text { если }(x, t) \in Q \backslash \bar{Q}_{\tau} .\end{cases}
$$

Здесь $\eta(t) \not \equiv 0$ - гладкая функция такая, что $0 \leqslant \eta(t) \leqslant 1$, причем $\eta(t) \equiv 0$ на $[0, \tau]=\left[0, t_{1}\right]$. Тогда $l(h)=\int_{0}^{T} h(x, t) d \mu(t)=0, \operatorname{Ker} l(h)=L_{2}(\Omega)$ и для любой $f(x) \in L_{2}(\Omega)$ функция $u(x, t ; f) \equiv 0$ в $\bar{Q}_{\tau}$ как решение задачи (6.1), (6.2). При этом, однако, $u(x, t ; f) \not \equiv 0$ в $Q \backslash \bar{Q}_{\tau}$, если $f(x) \not \equiv 0$ в $\Omega$.

Если в условиях теоремы 6 усилить требования на функцию $\widetilde{h}(x, t)$, то относительно функции $u(x, t)$ можно утверждать большее. В связи с этим дадим определение, аналогичное определению 3. 
ОПРЕДЕЛЕНИЕ 5. Будем говорить, что функция $\widetilde{h}(x, t)$ не изменяет знак по $t \in[0, T]$ в усиленном смысле, если она удовлетворяет определению 3 со строгими неравенствами для случаев 2 и 3.

Для функций, удовлетворяющих этому определению, введем функцию $\varphi_{0}(x)$ (“функцию знака" для $\widetilde{h}(x, t))$, как в определении 3 , причем по построению также справедливо тождество $\varphi_{0}^{2}(x) \equiv 1$ в $\Omega$.

Лемма 12. Пусть функиия $\widetilde{h}(x, t)$ непрерывна в норме $L_{\infty}(\Omega)$ и не изменяет знак по переменной $t \in[0, T]$ в усиленном смысле, функиия $\mu(t)$ удовлетворяет условию $(3.15)$, а $h(x, t):=\varphi_{0}(x) \widetilde{h}(x, t)$. Тогда $l(h)(x)=|l(\widetilde{h})(x)| u$ $\operatorname{Ker} l(h)=\operatorname{Ker} l(\widetilde{h})=\operatorname{Ker} h(\cdot, t)$ nри любом $t \in[0, T]$.

ДокАЗАТЕЛЬСТво. Для функции $\widetilde{h}(x, t)$ возможны следующие три случая:

1. $x_{0} \in \Omega_{0}$, и тогда $l(\widetilde{h})\left(x_{0}\right)=0$;

2. $x_{0} \in \Omega_{+}$, и тогда $l(\widetilde{h})\left(x_{0}\right)>0$, так как существует $t_{*} \in(0, T]-$ точка роста $\mu(t)$ такая, что $\widetilde{h}\left(x_{0}, t_{*}\right)>0$ (см. лемму 7 );

3. $x_{0} \in \Omega_{-}$, и тогда $l(\widetilde{h})\left(x_{0}\right)<0$ аналогично случаю 2 .

Таким образом, получим тождество $\varphi_{0}(x) \equiv \operatorname{sgn} l(\widetilde{h})(x)$ в $\Omega_{+} \cup \Omega_{-}$. Поскольку $\varphi_{0}^{2}(x) \equiv 1$, а неравенство $h(x, t) \geqslant 0$ в $Q$ следует из определения $\varphi_{0}(x)$, то $l(h)(x)=\varphi_{0}(x) l(\widetilde{h})(x) \equiv|l(\widetilde{h})(x)|$ в $\Omega$. Отсюда $\operatorname{Ker} l(h)=\operatorname{Ker}|l(\widetilde{h})|=\operatorname{Ker} l(\widetilde{h})$. Докажем, что $\operatorname{Ker} l(h)=\operatorname{Ker} h(\cdot, t)$ для всех $t \in[0, T]$, где через $h(\cdot, t)$ обозначен оператор умножения на функцию $h(x, t) \in L_{\infty}(\Omega)$ при фиксированном $t \in[0, T]$. Для любой точки $x_{0} \in \Omega$ возможны лишь следующие два случая:

1. либо $h\left(x_{0}, t\right) \equiv 0$ на $[0, T]$, и множество всех таких $x_{0} \in \Omega$ обозначим $\Omega_{1}$;

2. либо $h\left(x_{0}, t\right)>0$ на $[0, T]$, и множество всех таких $x_{0} \in \Omega$ обозначим $\Omega_{2}$. Очевидно, $\Omega=\Omega_{1} \cup \Omega_{2}$ и $l(h)(x)>0$ на $\Omega_{2}$. Функция $f \in \operatorname{Ker} l(h)$ только тогда, когда $f(x) \equiv 0$ на $\Omega_{2}$. Для таких функций $h(x, t) f(x) \equiv 0$ в $\Omega$ при любом $t \in[0, T]$. Обратно, пусть $f \in \operatorname{Ker} h(\cdot, t)$ при некотором $t \in[0, T]$. Тогда $f(x) \equiv 0$ на $\Omega_{2}$ и $f \in \operatorname{Ker} l(h)$. Лемма доказана.

Дадим теперь следствие из теоремы 6 при этих дополнительных условиях на функцию $\widetilde{h}(x, t)$.

СЛЕДСТВИЕ 7. Пусть выполнены все условия теоремы 6, причем функиия $\widetilde{h}(x, t)$ не изменяет знак по $t \in[0, T]$ в усиленном смысле, и рассмотрим задачу (6.1)-(6.3) с функцией $h(x, t) \equiv \varphi_{0}(x) \widetilde{h}(x, t)$. Пара $\{u ; f\} \in \operatorname{Ker}$ только тогда, когда $f \in \operatorname{Ker} l(h)$, при этом $u(x, t) \equiv 0$ во всем иилиндре $Q$.

ДокАзАТЕЛЬство. Необходимость. Если пара $\{u(x, t) ; f(x)\}-$ решение однородной обратной задачи (6.1)-(6.3), то, повторяя рассуждения доказательства первого утверждения в теореме 6 , получим $f^{ \pm} \in \operatorname{Ker} l(h)$, а из леммы 12 $h(x, t) f^{ \pm}(x) \equiv 0$ и $h(x, t) f(x) \equiv 0$ в $\Omega$ при любом $t \in[0, T]$. Из единственности решения прямой задачи (6.1), (6.2) с $h(x, t) f(x) \equiv 0$ в $Q$ получим $u(x, t) \equiv 0$ в $Q$. Необходимость доказана.

Достаточность. Пусть $f \in \operatorname{Ker} l(h)$. Тогда $f^{ \pm} \in \operatorname{Ker} l(h)$. Повторив рассуждения, приведенные при доказательстве второго утверждения теоремы 6 , получим, что решение задачи $(6.1),(6.2)$ с этим $f$ удовлетворяет (6.3). По лемме 12 имеем $f^{ \pm} \in \operatorname{Ker} h(\cdot, t)$, а тогда $h(x, t) f^{ \pm}(x) \equiv 0$ и $h(x, t) f(x) \equiv 0$ в $\Omega$ 
при всех $t \in[0, T]$, т.е. $h(x, t) f(x) \equiv 0$ в $Q$. Из единственности решения прямой задачи $(6.1),(6.2) u(x, t) \equiv 0$ в $Q$. Следствие доказано.

ЗАМЕчАНИЕ 11. В работе [35] понятие ядра было введено для граничной обратной задачи. В ней восстанавливалось граничное условие третьего рода при финальном или интегральном наблюдении, заданном на границе области $\Omega$, и было получено описание ядра задачи, аналогичное теореме 6 .

\section{§ 8. Полнота и базисность некоторых систем функций, связанных с обратной задачей}

8.1. Постановка задачи и вывод основного соотношения. При исследовании задачи особый интерес представляет получение необходимых и достаточных условий ее разрешимости. Для задачи (2.4)-(2.6) удается привести необходимые и достаточные условия единственности и корректности, связанные (соответственно) с полнотой и базисностью некоторой системы функций. Относительно эллиптического оператора при этом делается ряд дополнительных предположений. В этом параграфе рассмотрим обратную задачу нахождения пары функций $\{u(x, t) ; f(x)\}$ из следующих условий:

$$
\begin{gathered}
u_{t}(x, t)-L_{0} u(x, t)=h(x, t) f(x), \quad(x, t) \in Q, \\
u(x, 0)=0, \quad x \in \Omega, \quad B u(x, t)=0, \quad(x, t) \in S, \\
l(u)=\chi(x), \quad x \in \Omega .
\end{gathered}
$$

Здесь $\chi \in W_{2}^{2}(\Omega), B \chi(x)=0$ на $\partial \Omega$, а $L_{0}$ - стационарный, равномерно эллиптический (симметрический) оператор вида

$$
L_{0} u=\sum_{i, j=1}^{n} \frac{\partial}{\partial x_{i}}\left(a_{i j}(x) \frac{\partial u}{\partial x_{j}}\right)+c(x) u
$$

с коэффициентами $a_{i j} \in C^{1}(\bar{\Omega}), c \in L_{\infty}(\Omega)$ и выполняется условие $(\mathrm{E})$. Оператор граничных условий $B$ такой же, как в (2.2). Все эти условия будем считать выполненными всюду до конца параграфа. В отличие от задачи $(2.4)-(2.6)$, не предполагаем заранее, что $l(h)(x) \equiv 1$ в $\Omega$. Требования на функцию $h(x, t)$ в соответствующих утверждениях будем формулировать всякий раз отдельно.

Собственные функции и собственные значения задачи

$$
-L_{0} v(x)=\lambda v(x), \quad x \in \Omega, \quad B v(x)=0, \quad x \in \partial \Omega
$$

обозначим $\left\{e_{k}(x)\right\}$ и $\left\{\lambda_{k}\right\}$, занумеровав $\lambda_{k}$ в порядке возрастания модуля (с учетом кратности) и считая, что $\left\|e_{k}\right\|_{2, \Omega}=1$ для всех $k=1,2, \ldots$ Известно (см. [25], [31], [36]), что $e_{k} \in W_{2}^{2}(\Omega), \lambda_{k} \in \mathbb{R}, \lambda_{k} \rightarrow+\infty$. В нашем случае $\lambda_{1}>0$, а система $\left\{e_{k}(x)\right\}$ образует ортонормированный базис в $L_{2}(\Omega)$. Введем систему функций

$$
\psi_{k}(x):=\lambda_{k} \int_{0}^{T}\left(\int_{0}^{t} e^{-\lambda_{k}(t-\tau)} h(x, \tau) d \tau\right) d \mu(t) e_{k}(x) \equiv \beta_{k}(x) e_{k}(x), \quad k \in \mathbb{N} .
$$

Отметим, что функции $\beta_{k}(x) \stackrel{L_{\infty}(\Omega)}{\longrightarrow} l(h)(x)$ при $k \rightarrow \infty$, где символом $\stackrel{L_{\infty}(\Omega)}{\longrightarrow}$ обозначена сходимость по норме $L_{\infty}(\Omega)$. Точнее, справедлива следующая 
Лемма 13. Пусть $\mu \in \mathrm{BV}[0, T], \mu(0)=\mu(0+)$, функиия $h \in L_{\infty}(Q)$ и непрерывна по переменной $t \in[0, T]$ в норме $L_{\infty}(\Omega)$, а последовательность комплексных чисел $\left\{\lambda_{k}\right\}$ удовлетворяет условию

$$
\operatorname{Re} \lambda_{k} \rightarrow+\infty \operatorname{ma\kappa }, \text { umo }\left|\operatorname{Im} \lambda_{k}\right| \leqslant \operatorname{const} \operatorname{Re} \lambda_{k} .
$$

Тогда

$$
\beta_{k}(x) \stackrel{L_{\infty}(\Omega)}{\longrightarrow} l(h)(x)=\int_{0}^{T} h(x, t) d \mu(t) \quad n p u \quad k \rightarrow \infty .
$$

ДокАЗАтельство. Введем последовательность функций

$$
\Delta_{k}(x ; t):=\lambda_{k} \int_{0}^{t} \exp \left(-\lambda_{k}(t-\tau)\right) h(x, \tau) d \tau-h(x, t) .
$$

Докажем, что $l\left(\Delta_{k}\right)(x):=\int_{0}^{T} \Delta_{k}(x, t) d \mu(t) \stackrel{L_{\infty}(\Omega)}{\longrightarrow} 0$ при $k \rightarrow \infty$. Для любого $t \in[0, T]$ справедливо неравенство

$$
\begin{aligned}
\left\|\Delta_{k}(x ; t)\right\|_{\infty, \Omega} & \leqslant\left|\lambda_{k}\right| \int_{0}^{t} \exp \left(-\operatorname{Re} \lambda_{k}(t-\tau)\right)\|h(x, \tau)\|_{\infty, \Omega} d \tau+\|h(x, t)\|_{\infty, \Omega} \\
& \leqslant \max _{\tau \in[0, T]}\left\{\|h(x, \tau)\|_{\infty, \Omega}\right\} \cdot\left[\frac{\left|\lambda_{k}\right|}{\operatorname{Re} \lambda_{k}}\left(1-\exp \left(-t \operatorname{Re} \lambda_{k}\right)\right)+1\right] \\
& \leqslant \max _{\tau \in[0, T]}\left\{\|h(x, \tau)\|_{\infty, \Omega}\right\} \cdot\left(\frac{\left|\lambda_{k}\right|}{\operatorname{Re} \lambda_{k}}+1\right) \leqslant C_{1}
\end{aligned}
$$

при всех $k \in \mathbb{N}$ с некоторой константой $C_{1}>0$. Здесь использована оценка $\left|\operatorname{Im} \lambda_{k}\right| \leqslant$ const $\operatorname{Re} \lambda_{k}$, из которой следует ограниченность последовательности $\left|\lambda_{k}\right| / \operatorname{Re} \lambda_{k}$ (считаем при этом, что $\left.\operatorname{Re} \lambda_{k}>0\right)$.

Рассмотрим произвольное $\varepsilon>0$ и фиксируем его. По числу $\varepsilon /\left(2 C_{1}\right)$ из условия непрерывности функции $\mu(t)$ при $t=0$ справа найдем $\delta_{0}(\varepsilon)>0$ такое, что справедливо неравенство $\bigvee_{0}^{\delta_{0}}(\mu)<\varepsilon /\left(2 C_{1}\right)$ (см. [23; гл. 8, §1, теорема 1]). Тогда при всех $k \in \mathbb{N}$ получим

$$
\begin{aligned}
& \left\|\int_{0}^{T} \Delta_{k}(x ; t) d \mu(t)\right\|_{\infty, \Omega} \leqslant \int_{0}^{\delta_{0}}\left\|\Delta_{k}(x ; t)\right\|_{\infty, \Omega} d \nu(t)+\left\|\int_{\delta_{0}}^{T} \Delta_{k}(x ; t) d \mu(t)\right\|_{\infty, \Omega} \\
& \leqslant C_{1} \bigvee_{0}^{\delta_{0}}(\mu)+\left\|\int_{\delta_{0}}^{T} \Delta_{k}(x ; t) d \mu(t)\right\|_{\infty, \Omega}<\frac{\varepsilon}{2}+\left\|\int_{\delta_{0}}^{T} \Delta_{k}(x ; t) d \mu(t)\right\|_{\infty, \Omega},
\end{aligned}
$$

где $\nu(t):=\bigvee_{0}^{t}(\mu)$. Для второго слагаемого в (8.7) справедлива оценка

$$
\left\|\int_{\delta_{0}}^{T} \Delta_{k}(x ; t) d \mu(t)\right\|_{\infty, \Omega} \leqslant \max _{\left[\delta_{0}, T\right]}\left\{\left\|\Delta_{k}(x ; t)\right\|_{\infty, \Omega}\right\} \bigvee_{\delta_{0}}^{T}(\mu) \leqslant C_{0} \max _{\left[\delta_{0}, T\right]}\left\{\left\|\Delta_{k}(x ; t)\right\|_{\infty, \Omega}\right\}
$$

в которой $C_{0}:=\bigvee_{0}^{T}(\mu)$. Если $C_{0}=0$, то утверждение леммы очевидно, поэтому считаем ниже $C_{0}>0$. Докажем, что $\left\|\Delta_{k}(x, t)\right\|_{\infty, \Omega} \stackrel{\left[\delta_{0}, T\right]}{\rightrightarrows} 0$ при $k \rightarrow \infty$. Для 
этого $\Delta_{k}(x, t)$ преобразуем к виду

$$
\begin{aligned}
\Delta_{k}(x ; t) & =\lambda_{k} \int_{0}^{t} \exp \left(-\lambda_{k}(t-\tau)\right)[h(x, \tau)-h(x, t)] d \tau-\exp \left(-\lambda_{k} t\right) h(x, t) \\
& =I(x, t ; k)-\exp \left(-\lambda_{k} t\right) h(x, t)=I_{1}(x, t ; k)+I_{2}(x, t ; k)-\exp \left(-\lambda_{k} t\right) h(x, t)
\end{aligned}
$$

при всех $t \in\left[\delta_{0}, T\right]$. Слагаемые $I_{1}$ и $I_{2}$ определяются формулами

$$
\begin{aligned}
& I_{1}(x, t ; k):=\lambda_{k} \int_{0}^{t-\delta} e^{\lambda_{k}(t-\tau)} g(x, t, \tau) d \tau \\
& I_{2}(x, t ; k):=\lambda_{k} \int_{t-\delta}^{t} e^{\lambda_{k}(t-\tau)} g(x, t, \tau) d \tau
\end{aligned}
$$

функция $g(x, t, \tau):=h(x, \tau)-h(x, t)$, а положительное число $\delta<\delta_{0}$ выберем ниже. Последнее слагаемое в $\Delta_{k}(x, t)$ легко оценивается на отрезке $\left[\delta_{0}, T\right]$

$$
\max _{\left[\delta_{0}, T\right]}\left\{\left\|e^{-\lambda_{k} t} h(x, t)\right\|_{\infty, \Omega}\right\} \leqslant e^{-\delta_{0} \operatorname{Re} \lambda_{k}}\|h(x, t)\|_{\infty, Q}
$$

поэтому его норма в $L_{\infty}(\Omega)$ равномерно на $\left[\delta_{0}, T\right]$ стремится к нулю при $k \rightarrow \infty$. Выберем $N_{1}(\varepsilon) \in \mathbb{N}$ так, чтобы $\exp \left(-\delta_{0} \operatorname{Re} \lambda_{k}\right)\|h(x, t)\|_{\infty, Q}<\varepsilon /\left(6 C_{0}\right)$ при всех $k \geqslant N_{1}$.

Оценим теперь слагаемое $I_{2}(x, t ; k)$. По условию функция $h(x, t)$ равномерно непрерывна на $[0, T]$ в норме $L_{\infty}(\Omega)$, откуда следует, что

$$
\forall \widetilde{\varepsilon}>0 \quad \exists \delta(\widetilde{\varepsilon})>0: \quad\|g\|_{\infty, \Omega} \equiv\|h(x, \tau)-h(x, t)\|_{\infty, \Omega}<\widetilde{\varepsilon} \quad \forall \tau \in[t-\delta, t] .
$$

Тогда для всех $t$, принадлежащих отрезку $\left[\delta_{0}, T\right]$, справедливо неравенство

$$
\left\|I_{2}(x, t ; k)\right\|_{\infty, \Omega} \leqslant \widetilde{\varepsilon} \cdot \frac{\left|\lambda_{k}\right|}{\operatorname{Re} \lambda_{k}} \cdot\left(1-\exp \left(-\delta \operatorname{Re} \lambda_{k}\right)\right) \leqslant C_{2} \widetilde{\varepsilon} \quad \text { при всех } k \in \mathbb{N} .
$$

По $\widetilde{\varepsilon}=\varepsilon /\left(6 C_{2} C_{0}\right)$, выбирая $\delta=\delta(\widetilde{\varepsilon}) \in\left(0, \delta_{0}\right)$ из определения равномерной непрерывности $h(x, t)$ в норме $L_{\infty}(\Omega)$ на $[0, T]$, получим, что

$$
\max _{t \in\left[\delta_{0}, T\right]}\left\{\left\|I_{2}(x, t ; k)\right\|_{\infty, \Omega}\right\}<\frac{\varepsilon}{6 C_{0}}, \quad k \in \mathbb{N} .
$$

Оценим слагаемое $I_{1}(x, t ; k)$ для всех $t \in\left[\delta_{0}, T\right]$ в норме $L_{\infty}(\Omega)$ :

$$
\begin{aligned}
\left\|I_{1}(x, t ; k)\right\|_{\infty, \Omega} & \leqslant\left|\lambda_{k}\right| \int_{0}^{t-\delta} \exp \left(-\operatorname{Re} \lambda_{k}(t-\tau)\right)\|h(x, \tau)-h(x, t)\|_{\infty, \Omega} d \tau \\
& \leqslant 2\|h(x, t)\|_{\infty, Q} \frac{\left|\lambda_{k}\right|}{\operatorname{Re} \lambda_{k}} \cdot\left(\exp \left(-\delta \operatorname{Re} \lambda_{k}\right)-\exp \left(-t \operatorname{Re} \lambda_{k}\right)\right) \\
& \leqslant 2\|h(x, t)\|_{\infty, Q} \frac{\left|\lambda_{k}\right|}{\operatorname{Re} \lambda_{k}} \exp \left(-\delta \operatorname{Re} \lambda_{k}\right) .
\end{aligned}
$$

Правая часть в этой оценке стремится к нулю при $k \rightarrow \infty$. Отсюда найдется номер $N_{2}(\varepsilon) \in \mathbb{N}$ такой, что $\max \left\{\left\|I_{1}(x, t ; k)\right\|_{\infty, \Omega} \mid t \in\left[\delta_{0}, T\right]\right\}<\varepsilon /\left(6 C_{0}\right)$ при 
всех $k \geqslant N_{2}(\varepsilon)$. Тогда для всех натуральных $k \geqslant \max \left(N_{1}, N_{2}\right)$, используя (8.7) и (8.8), получим, что выполняется неравенство

$$
\left\|\int_{0}^{T} \Delta_{k}(x ; t) d \mu(t)\right\|_{\infty, \Omega}<\frac{\varepsilon}{2}+\frac{\varepsilon}{6 C_{0}} 3 C_{0}=\varepsilon .
$$

Лемма доказана.

Система (8.6) тесно связана с исследуемой обратной задачей. Впервые эта связь отмечалась в работе [8] для задач с финальным и интегральным наблюдением (по этому поводу см. также [20]). Она основывается на следующем соотношении, справедливом для любого решения $u(x, t ; f)$ прямой задачи $(8.1)$, (8.2), в предположении, что $h, h_{t} \in L_{\infty, 2}(Q)$ :

$$
-\left(L_{0} l(u), e_{k}\right)=\left(f, \psi_{k}\right) \quad \text { при } \quad k=1,2, \ldots .
$$

Здесь $\left(g_{1}, g_{2}\right):=\int_{\Omega} g_{1}(x) g_{2}(x) d x-$ скалярное произведение в $L_{2}(\Omega)$. Для получения (8.9) фиксируем $f \in L_{2}(\Omega)$ и решим задачу (8.1), (8.2) с этим $f$. По нашему предположению $h f, h_{t} f \in L_{2}(Q)$, а тогда решение прямой задачи $u \in W_{2}^{2,1}(Q)$ и обладает дополнительной гладкостью (см. леммы 2,3 ) $u_{t}(\cdot, t) \in C\left([0, T] ; L_{2}(\Omega)\right), u(\cdot, t) \in C\left([0, T] ; W_{2}^{2}(\Omega)\right)$, в силу чего законны все последующие выкладки по выводу (8.9). Рассмотрим равенство (8.1) при $t=\tau$. Умножим обе его части на $e^{\lambda_{k} \tau} e_{k}(x)$ скалярно в $L_{2}(\Omega)$ и проинтегрируем по $\tau \in[0, t]$ с учетом условий $(8.2)$

$$
\begin{aligned}
& e^{\lambda_{k} t}\left(u(\cdot, t), e_{k}\right)-\int_{0}^{t} e^{\lambda_{k} \tau}\left(L_{0} u(\cdot, \tau), e_{k}\right) d \tau \\
& \quad-\lambda_{k} \int_{0}^{t} e^{\lambda_{k} \tau}\left(u(\cdot, \tau), e_{k}\right) d \tau=\int_{0}^{t} e^{\lambda_{k} \tau}\left(h(\cdot, \tau) f, e_{k}\right) d \tau
\end{aligned}
$$

Воспользуемся замкнутостью, "стационарностью" и симметричностью оператора $L_{0}$ с областью определения $D\left(L_{0}\right)=\left\{v \in W_{2}^{2}(\Omega) \mid B v(x)=0, x \in \partial \Omega\right\}$. Интегрируя второе слагаемое по частям по $x$, выведем равенство

$$
\left(u(\cdot, t), e_{k}\right)=\left(f, \int_{0}^{t} e^{-\lambda_{k}(t-\tau)} h(\cdot, \tau) d \tau e_{k}\right) .
$$

Умножая его на $\lambda_{k}$ и интегрируя с мерой $d \mu(t)$ по $[0, T]$, получим

$$
\left(l(u), \lambda_{k} e_{k}\right)=\left(f, \psi_{k}\right) \quad \text { при } k=1,2, \ldots
$$

Подставляя $\lambda_{k} e_{k}(x)=-L_{0} e_{k}(x), x \in \Omega$, в левую часть (8.10) и интегрируя дважды по частям с учетом $B e_{k}(x)=0, x \in \partial \Omega$, выводим (8.9).

\section{2. Связь полноты системы (8.6) с единственностью решения об-} ратной задачи. Из установленных соотношений получим, что полнота системы $\left\{\psi_{k}(x)\right\}$ эквивалентна единственности решения обратной задачи. Здесь под полнотой системы $\left\{\psi_{k}(x)\right\}$ в $L_{2}(\Omega)$, как обычно, понимается отсутствие ненулевого элемента $L_{2}(\Omega)$, ортогонального всем элементам $\left\{\psi_{k}(x)\right\}$. Отметим, что сведение обратной задачи к степенной проблеме моментов использовалась в работе [37; гл. $6, \S 1]$, но для обратной задачи другого, чем здесь, вида. Имеет место 
Tеорема 7. Пусть $h, h_{t} \in L_{\infty, 2}(Q)$, а операторы $L_{0}$ и B удовлетворяют условиям, перечисленным в начале §8. Система $\left\{\psi_{k}(x)\right\}$, введенная в (8.6), полна в $L_{2}(\Omega)$ только тогда, когда решение обратной задачи (8.1)-(8.3) единственно.

ДоказАтельство. Необходимость. Пусть $\left\{\psi_{k}(x)\right\}$ полна. Рассмотрим пару функций $\{u(x, t) ; f(x)\}$ - решение однородной обратной задачи $(8.1),(8.2)$ с $l(u)=0$ в условии (8.3). С этим $f$ повторим вывод (8.9) и получим, что при всех $k \in \mathbb{N}$ выполняются равенства $\left(f, \psi_{k}\right)=0$. Отсюда в силу полноты $f=\theta$, а тогда из единственности решения прямой задачи (8.1), (8.2) следует, что $u=0$ в $Q$.

Достаточность. Пусть решение обратной задачи (8.1)-(8.3) единственно, а система $\left\{\psi_{k}(x)\right\}$ не является полной. Тогда найдется элемент $f \neq \theta$, ортогональный системе $\left\{\psi_{k}(x)\right\}$. С этим $f$ решим задачу (8.1), (8.2) и получим функцию $u(x, t ; f)$, которая будет удовлетворять (8.9), т.е. равенствам $-\left(L_{0} l(u), e_{k}\right)=\left(f, \psi_{k}\right)=0$ при всех $k \in \mathbb{N}$. Так как $\left\{e_{k}(x)\right\}-$ это базис, то отсюда получим, что $-L_{0} l(u)(x)=0$ при $x \in \Omega$. Поскольку $u(\cdot, t) \in C\left([0, T] ; W_{2}^{2}(\Omega)\right)$ и $B u(x, t)=0,(x, t) \in S$, то $B l(u)(x)=0, x \in \partial \Omega$. Из единственности решения краевой задачи для эллиптического уравнения следует, что $l(u)(x)=0$ в $\Omega$, т.е. пара функций $\{u(x, t) ; f(x)\}$ - нетривиальное решение однородной обратной задачи, а это противоречит единственности ее решения. Теорема 7 доказана.

Как следствие получаем достаточные условия полноты системы $\left\{\psi_{k}(x)\right\}$.

СлеДСтвиЕ 8. Пусть выполнены условия теоремы 7, функиия $\mu(t)$ удовлетворяет $(3.15), h(x, t) \geqslant 0$ в $Q$, a $l(h)(x)>0$ в $\Omega$. Предположим дополнительно, что выполняется хотя бы одно из условий:

1. $h_{t}(x, t) \geqslant 0$ в $Q$;

2. $d \mu(t)=\omega(t) d t$ с функиией $\omega \in \mathrm{BV}[0, T]$, которая является невозрастающей на $[0, T]$.

Тогда система $\left\{\psi_{k}(x)\right\}$ полна в $L_{2}(\Omega)$.

ДокАзАтЕЛЬство. Оператор $L_{0}$ и все заданные функции удовлетворяют достаточным условиям единственности решения обратной задачи, сформулированным в теореме 3. По теореме 7 получаем полноту системы функций $\left\{\psi_{k}(x)\right\}$. Следствие доказано.

8.3. Проблема моментов. Связь корректности обратной задачи с базисностью системы (8.6). Для системы $\left\{\psi_{k}\right\}$ может быть поставлена проблема моментов. Найти функцию $f(x) \in L_{2}(\Omega)$, удовлетворяющую равенствам

$$
\left(\psi_{k}, f\right):=\int_{\Omega} \psi_{k}(x) f(x) d x=\alpha_{k} \quad \text { при всех } k=1,2, \ldots,
$$

где числовая последовательность $\alpha=\left\{\alpha_{k}\right\}$ задана. Условие $\alpha \in l_{2}$ означает, как обычно, что $\sum_{k}\left|\alpha_{k}\right|^{2}<\infty$. В работе [32] доказано, что разрешимость обратной задачи с финальным наблюдением эквивалентна разрешимости соответствующей проблемы моментов. Для более общей системы функций, определенной в (8.6), справедлив аналогичный результат. 
Теорема 8. Пусть выполнены условия теоремы 7. Разрешимость проблемы моментов (8.11) эквивалентна разрешимости обратной задачи (8.1)-(8.3), а именно справедливы следующие два утверждения.

1. Пусть последовательность $\alpha \in l_{2}$ и (8.11) разрешима. Тогда функция

$$
\chi(x):=\sum_{k} \frac{\alpha_{k}}{\lambda_{k}} e_{k}(x) \in W_{2}^{2}(\Omega), \quad \text { причем } B \chi(x)=0, \quad x \in \partial \Omega,
$$

и обратная задача (8.1)-(8.3) разрешима.

2. Пусть обратная задача (8.1)-(8.3) с $l(u)=\chi(x) \in W_{2}^{2}(\Omega)$ и такой, что $B \chi(x)=0, x \in \partial \Omega$, разрешима. Тогда (8.11) с $\alpha_{k}=\lambda_{k}\left(\chi, e_{k}\right)$ разрешима и последовательность $\alpha \in l_{2}$.

Доказательство. Рассмотрим $f \in L_{2}(\Omega)$ - решение проблемы моментов (8.11) и введем функцию $u(x, t ; f)$ как решение $(8.1),(8.2)$ с этим $f$. Тогда из (8.10) имеем $\lambda_{k}\left(l(u), e_{k}\right)=\alpha_{k}$. В силу свойства дополнительной гладкости решений прямой задачи (см. леммы 2,3$)$ имеем $l(u) \in W_{2}^{2}(\Omega)$, причем $B l(u)(x)=0, x \in \partial \Omega$. Но $\left\{e_{k}(x)\right\}$ - ортонормированный базис в $L_{2}(\Omega)$, поэтому справедливо разложение $l(u)(x)=\sum_{k}\left(\alpha_{k} / \lambda_{k}\right) e_{k}(x)=\chi(x)$. Первое утверждение доказано.

Пусть пара функций $\{u(x, t) ; f(x)\}$ - решение обратной задачи (8.1)-(8.3) с функцией $\chi \in W_{2}^{2}(\Omega)$. Из условия согласования $B \chi(x)=0$ на $\partial \Omega$. Тогда в силу (8.10) при всех $k \in \mathbb{N}$ выполнены равенства $\left(f, \psi_{k}\right)=\lambda_{k}\left(\chi, e_{k}\right)$, т.е. проблема моментов разрешима. В обозначениях теоремы $\alpha_{k}=\lambda_{k}\left(\chi, e_{k}\right)$. Докажем, что последовательность $\alpha \in l_{2}$. Последнее следует из цепочки равенств

$$
\begin{aligned}
\sum_{k=1}^{N}\left|\alpha_{k}\right|^{2} & =\left\|\sum_{k=1}^{N} \alpha_{k} e_{k}(x)\right\|^{2}=\left\|\sum_{k=1}^{N} \lambda_{k}\left(\chi, e_{k}\right) e_{k}(x)\right\|^{2} \\
& =\left\|\sum_{k=1}^{N}\left(-L_{0} \chi, e_{k}\right) e_{k}(x)\right\|^{2} \rightarrow\left\|L_{0} \chi(x)\right\|^{2} \quad \text { при } N \rightarrow \infty,
\end{aligned}
$$

где $\|\cdot\|$ - норма в $L_{2}(\Omega)$ и использовано, что $\chi \in W_{2}^{2}(\Omega)$ и $B \chi(x)=0, x \in \partial \Omega$. Теорема доказана.

При некоторых условиях для системы $\left\{\psi_{k}(x)\right\}$ существует биортогональная.

Теорема 9. Пусть при всех $k \in \mathbb{N}$ обратная задача (8.1)-(8.3) разрешима c $l(u)=\left(1 / \lambda_{k}\right) e_{k}(x)$ в условии наблюдения (8.3). Тогда существует биортогональная $\kappa\left\{\psi_{k}(x)\right\}$ система $\left\{g_{k}(x)\right\}$. Если решение обратной задачи единственно, то и $\left\{g_{k}(x)\right\}$ единственна.

ДокАЗАТЕЛьство. По условию теоремы существует последовательность пар функций $\left\{u_{n}(x, t) ; g_{n}(x)\right\}$, являющихся решениями обратных задач $(8.1)$, (8.2) с соответствующими условиями наблюдения $l\left(u_{n}\right)(x)=e_{n}(x) / \lambda_{n}$. Для этих функций выполняется соотношение (8.10): $\lambda_{k}\left(l\left(u_{n}\right), e_{k}\right)=\left(g_{n}, \psi_{k}\right)$, откуда следует $\left(g_{n}, \psi_{k}\right)=\left(\lambda_{k} / \lambda_{n}\right)\left(e_{k}, e_{n}\right)=\delta_{n k}$, т.е. $\left\{g_{n}(x)\right\}$ - биортогональная система. Если решение указанных обратных задач единственно, то и система $\left\{g_{n}(x)\right\}$ единственна. Теорема 9 доказана. 
ОПРЕДЕЛЕНиЕ 6. Обратная задача (8.1)-(8.3) называется корректной, если для любой функции $\chi(x) \in W_{2}^{2}(\Omega)$ такой, что $B \chi(x)=0$ на $\partial \Omega$, существует единственная функция $f(x) \in L_{2}(\Omega)$, для которой решение $u(x, t ; f)$ прямой задачи (8.1), (8.2) удовлетворяет (8.3) и при этом справедлива оценка устойчивости

$$
\|f\|_{2, \Omega} \leqslant C\left\|L_{0} \chi\right\|_{2, \Omega} .
$$

Теорема 10. Пусть выполнены условия теоремы 7. Система $\left\{\psi_{k}(x)\right\}$, определенная в (8.6), образует базис Рисса в $L_{2}(\Omega)$ только тогда, когда обратная задача (8.1)-(8.3) корректна.

ДокАЗАТЕЛЬСтво. При доказательстве этой теоремы для краткости введем обозначения $H:=L_{2}(\Omega), H_{1}:=W_{2}^{2}(\Omega)$ - гильбертовы пространства с соответствующими нормами. Множество $D\left(L_{0}\right):=\left\{\chi(x) \in W_{2}^{2}(\Omega) \mid B \chi(x)=0, x \in \partial \Omega\right\}$ является замкнутым в норме $W_{2}^{2}(\Omega)$.

Необходимость. Введем оператор $G: H \rightarrow H$ по правилу $G f:=\sum_{k}\left(f, \psi_{k}\right) e_{k}$. Предположим, что обратная задача корректна. Из тождества (8.9) имеем $\left(f, \psi_{k}\right)=\left(-L_{0} l(u), e_{k}\right) \in l_{2}$. Отсюда следует, что $G-$ линейный оператор и его область определения $D(G)=H$. В силу свойств дополнительной гладкости решения задачи (8.1), (8.2) (см. лемму 3$)$, функция $u=u(x, t ; f) \in C\left([0, T] ; H_{1}\right)$ и $l(u) \in D\left(L_{0}\right)$. Из цепочки неравенств

$$
\|G f\|=\left\|L_{0} l(u)\right\| \leqslant \text { const } \max _{t \in[0, T]}\left\{\|u(\cdot, t)\|_{2, \Omega}^{(2)}\right\} \leqslant \text { const }\|f\|
$$

получим, что $G \in \mathscr{L}(H)$. Докажем теперь, что $R(G)=H$, т.е. область значений совпадает с $H$. Рассмотрим произвольный элемент $g \in H$ и разложим его по базису $\left\{e_{k}\right\}: g=\sum_{k} \alpha_{k} e_{k}$, где $\alpha_{k}=\left(g, e_{k}\right), \alpha \in l_{2}$. Отметим, что $e_{k} \in D\left(L_{0}\right)$ при всех $k \in \mathbb{N}$. Возьмем последовательность функций

$$
\chi_{n}(x):=\sum_{k=1}^{n} \frac{\alpha_{k}}{\lambda_{k}} e_{k}(x) \in D\left(L_{0}\right) .
$$

Имеем

$$
-L_{0} \chi_{n}=\sum_{k=1}^{n} \alpha_{k} e_{k} \stackrel{H}{\longrightarrow} g, \quad n \rightarrow \infty .
$$

Из второго основного неравенства для эллиптических операторов справедлива оценка $\left\|\chi_{n}\right\|_{2, \Omega}^{(2)} \leqslant C\left\|-L_{0} \chi_{n}\right\|$. Поэтому сходятся последовательности $\chi_{n} \stackrel{H_{1}}{\longrightarrow} \chi$ и $-L_{0} \chi_{n} \stackrel{H}{\longrightarrow}-L_{0} \chi=g$ при $n \rightarrow \infty$. Решив обратную задачу (8.1)-(8.3) с этой функцией $\chi \in D\left(L_{0}\right)$, получим элемент $f \in H$, который удовлетворяет равенствам $\left(f, \psi_{k}\right)=\left(-L_{0} \chi, e_{k}\right)=\left(g, e_{k}\right)=\alpha_{k}$. По определению оператора $G$ будем иметь $G f=g$, т.е. $R(G)=H$. Докажем, что $\operatorname{Ker} G=\{\theta\}$. Уравнение $G f=\theta$ равносильно равенствам $\left(f, \psi_{k}\right)=0$ при всех $k \in \mathbb{N}$. Отсюда в силу полноты системы $\left\{\psi_{k}(x)\right\}$ (см. теорему 7 ) имеем $f=\theta$. Тогда определен обратный оператор $G^{-1}: R(G)=H \rightarrow D(G)=H$, а поскольку $G \in \mathscr{L}(H)$, то по теореме Банаха $G^{-1} \in \mathscr{L}(H)$ (см. [22; гл. $2, \S 2$, теорема 2]). Из определения оператора $G$ имеем $G g_{k}=e_{k}$, или $g_{k}=G^{-1} e_{k}$, для всех $k \in \mathbb{N}$, т.е. биортогональная система $\left\{g_{k}\right\}$ является базисом Рисса в $H$. Тогда и система $\left\{\psi_{k}(x)\right\}-$ тоже базис Рисса в $H$ (см. [38; гл. $6, \S \S 1,2])$. 
Достаточность. Пусть $\left\{\psi_{k}(x)\right\}$ - базис Рисса в $H$. Тогда существует биортогональная система $\left\{g_{k}\right\}$, которая также является базисом Рисса, и произвольный элемент $f \in H$ раскладывается по этому базису $f=\sum_{k=1}^{\infty}\left(f, \psi_{k}\right) g_{k}$. Существует оператор $U$ такой, что $U e_{k}=g_{k}$, причем $U, U^{-1} \in \mathscr{L}(H)$. Рассмотрим произвольную $\chi \in D\left(L_{0}\right)$ и докажем, что решение обратной задачи (8.1)-(8.3) с этой функцией существует, единственно и справедлива оценка устойчивости. Для этого возьмем числовую последовательность $\left\{\alpha_{k}\right\}:=\left\{\lambda_{k}\left(\chi, e_{k}\right)\right\} \in l_{2}$ и рассмотрим проблему моментов $\left(f, \psi_{k}\right)=\alpha_{k}, k \in \mathbb{N}$. Определим $f_{n}:=\sum_{k=1}^{n} \alpha_{k} g_{k}=$ $U\left(\sum_{k=1}^{n} \alpha_{k} e_{k}\right)$, тогда $f_{n} \stackrel{H}{\longrightarrow} f:=U(g)$, где $g=\sum_{k} \alpha_{k} e_{k}$. Для так построенного элемента $f \in H$ имеем $\left(f, \psi_{k}\right)=\alpha_{k}$, т.е. существует и притом единственное решение проблемы моментов, а тогда по теореме 8 и обратная задача однозначно разрешима. Оценка устойчивости следует из цепочки неравенств

$$
\|f\|_{H}^{2} \leqslant\|U\|^{2}\|g\|_{H}^{2}=\|U\|^{2} \sum_{k=1}^{\infty}\left|\alpha_{k}\right|^{2}=\|U\|^{2}\left\|L_{0} \chi\right\|_{H}^{2} .
$$

Теорема 10 доказана.

Из доказанных теорем 2 и 10 в качестве простого следствия получаем достаточные условия базисности системы (8.6).

СлеДСТвиЕ 9. Пусть выполнены условия теоремы 7, функиия $\mu(t)$ удовлетворяет (3.15) и справедливы неравенства $|l(h)(x)| \geqslant \delta>0$ в $\Omega, h^{0}(x, t) \geqslant 0$ в $Q$, где $h^{0}(x, t) \equiv h(x, t) / l(h)(x)$. Предположим дополнительно, что выполняется хотя бы одно из условий:

1. $h_{t}^{0}(x, t) \geqslant 0$ в $Q$;

2. $d \mu(t)=\omega(t) d t$ с функцией $\omega \in \mathrm{BV}[0, T]$, которая является невозрастающей на $[0, T]$.

Тогда система $\left\{\psi_{k}(x)\right\}$ образует базис Рисса в $L_{2}(\Omega)$.

ДокАзАтЕльство. Все заданные функции и операторы удовлетворяют достаточным условиям корректности обратной задачи (8.1)-(8.3), сформулированным в теореме 2. По теореме 10 получаем, что $\left\{\psi_{k}(x)\right\}$ является базисом Рисса в $L_{2}(\Omega)$. Следствие доказано.

8.4. Достаточные условия базисности по Бари системы (8.6). При соответствующих условиях на функцию $h(x, t)$ и размерность $n$ пространства $\mathbb{R}^{n}$ система $\left\{\psi_{k}(x)\right\}$ образует базис Бари в $L_{2}(\Omega)$, т.е. базис, квадратично близкий к ортонормированному. Для доказательства помимо леммы 13 нам понадобится следующее утверждение, уточняющее асимптотическое поведение последовательности функций $\beta_{k}(x)$ при $k \rightarrow \infty$.

ЛЕмма 14. Пусть $\mu(t) \in W_{\infty}^{1}[0, \delta] \cap \mathrm{BV}[0, T], \mu(0)=\mu(0+)$,

$$
h(x, t), h_{t}(x, t) \in L_{\infty}(Q), \quad \beta\left(x, t ; \lambda_{k}\right):=\lambda_{k} \int_{0}^{t} \exp \left(-\lambda_{k}(t-\tau)\right) h(x, \tau) d \tau,
$$

а последовательность комплексных чисел $\left\{\lambda_{k}\right\}$ такова, что $\operatorname{Re} \lambda_{k} \rightarrow+\infty$.

Тогда имеет место равенство

$$
\beta_{k}(x):=\int_{0}^{T} \beta\left(x, t ; \lambda_{k}\right) d \mu(t)=l(h)(x)+\alpha\left(x ; \lambda_{k}\right),
$$


где для функиии $\alpha\left(x ; \lambda_{k}\right)$ справедлива оченка $\left\|\alpha\left(x ; \lambda_{k}\right)\right\|_{\infty, \Omega} \leqslant$ const $/ \operatorname{Re} \lambda_{k}$.

ДокАЗАТЕльство. В силу условий леммы введенные функции $\beta\left(x, t ; \lambda_{k}\right)$ допускают представление (для п.в. $x \in \Omega$ ):

$$
\begin{aligned}
\beta\left(x, t ; \lambda_{k}\right) & =h(x, t)-h(x, 0) e^{-\lambda_{k} t}-\int_{0}^{t} \exp \left(-\lambda_{k}(t-\tau)\right) h_{\tau}^{\prime}(x, \tau) d \tau \\
& =h(x, t)-h(x, 0) e^{-\lambda_{k} t}-I\left(x, t ; \lambda_{k}\right) \\
\beta_{k}(x) & =l(\beta)\left(x ; \lambda_{k}\right)=\int_{0}^{T} \beta\left(x, t ; \lambda_{k}\right) d \mu(t) \\
& =l(h)(x)-h(x, 0) \int_{0}^{T} e^{-\lambda_{k} t} d \mu(t)-\int_{0}^{T} I\left(x, t ; \lambda_{k}\right) d \mu(t) .
\end{aligned}
$$

Оценим в последнем равенстве каждый из двух интегралов отдельно, считая $\operatorname{Re} \lambda_{k}>0$. Получим

$$
\begin{aligned}
& \left|\int_{0}^{T} e^{-\lambda_{k} t} d \mu(t)\right| \leqslant\left|\int_{0}^{\delta} e^{-\lambda_{k} t} d \mu(t)\right|+\left|\int_{\delta}^{T} e^{-\lambda_{k} t} d \mu(t)\right| \\
& \quad \leqslant \operatorname{essup}_{[0, \delta]}\left\{\left|\mu^{\prime}(t)\right|\right\} \frac{1}{\operatorname{Re} \lambda_{k}}\left(1-e^{-\delta \operatorname{Re} \lambda_{k}}\right)+e^{-\delta \operatorname{Re} \lambda_{k}} \bigvee_{\delta}^{T}(\mu) \leqslant \frac{\operatorname{const}}{\operatorname{Re} \lambda_{k}}, \quad(8.12) \\
& \left\|\int_{0}^{T} I\left(x, t ; \lambda_{k}\right) d \mu(t)\right\|_{\infty, \Omega}=\left\|\int_{0}^{T}\left(\int_{0}^{t} \exp \left(-\lambda_{k}(t-\tau)\right) h_{\tau}^{\prime}(x, \tau) d \tau\right) d \mu(t)\right\|_{\infty, \Omega} \\
& \quad \leqslant \max _{t \in[0, T]}\left\{\left\|\int_{0}^{t} \exp \left(-\lambda_{k}(t-\tau)\right) h_{\tau}^{\prime}(x, \tau) d \tau\right\|_{\infty, \Omega}\right\} \bigvee_{0}^{T}(\mu), \\
& \left\|\int_{0}^{t} \quad \exp _{0}\left(-\lambda_{k}(t-\tau)\right) h_{\tau}^{\prime}(x, \tau) d \tau\right\|_{\infty, \Omega} \leqslant \int_{0}^{t} \exp \left(-\operatorname{Re} \lambda_{k}(t-\tau)\right)\left\|h_{\tau}^{\prime}(x, \tau)\right\|_{\infty, \Omega} d \tau \\
& \leqslant \operatorname{essup}\left\{\left\|h_{\tau}^{\prime}(x, \tau)\right\|_{\infty, \Omega}\right\} \frac{1}{\operatorname{Re} \lambda_{k}}\left(1-e^{-t \operatorname{Re} \lambda_{k}}\right) \leqslant \frac{\operatorname{const}}{\operatorname{Re} \lambda_{k}}
\end{aligned}
$$

В неравенствах (8.12) и (8.14) одним и тем же символом const обозначены разные константы, зависящие от заданных функций.

Из (8.12)-(8.14) получим, что $\beta_{k}(x)=l(h)(x)+\alpha\left(x ; \lambda_{k}\right)$, где для последовательности функций $\alpha\left(x ; \lambda_{k}\right)$ справедлива оценка $\left\|\alpha\left(x ; \lambda_{k}\right)\right\|_{\infty, \Omega} \leqslant$ const $/ \operatorname{Re} \lambda_{k}$. Лемма 14 доказана.

ЗАмЕчАнИЕ 12. Доказательство леммы 14 не использует присутствующее в формулировке леммы 13 условие $\left|\operatorname{Im} \lambda_{k}\right| \leqslant \operatorname{const} \operatorname{Re} \lambda_{k}$. Но при этом, по сравнению с леммой 13 , накладываются дополнительные условия гладкости на функции $\mu(t)$ и $h(x, t)$.

Напомним, что в условиях этого параграфа собственные значения $\lambda_{k}$ задачи (8.5) положительны. Используя известную асимптотику $\lambda_{k}$ (см., например, [39], [26], [40])

$$
\lambda_{k} \sim \text { const } \cdot k^{2 / n} \quad \text { при } k \rightarrow \infty,
$$

установим следующее утверждение. 
СлЕДСтвиЕ 10. Пусть операторы $L_{0}$ и В удовлетворяют условиям, перечисленным в начале $\S 8, h, h_{t} \in L_{\infty}(Q), \mu(t) \in W_{\infty}^{1}[0, \delta]$ при некотором $\delta>0 u$ удовлетворяет (3.15), функиия $h(x, t) \geqslant 0$ в $Q$, a $l(h)(x) \equiv 1$ в $\Omega$. Предположим дополнительно, что выполняется хотя бы одно из условий:

1. $h_{t}(x, t) \geqslant 0$ в $Q$;

2. $d \mu(t)=\omega(t) d t$ с функцией $\omega \in \mathrm{BV}[0, T]$, которая является невозрастающей на $[0, T]$.

Тогда для размерностей $n=1,2,3$ система $\left\{\psi_{k}(x)\right\}$, определенная в $(8.6)$, образует базис Бари в $L_{2}(\Omega)$.

ДокАЗАТЕЛЬСтво. В силу лемм 13 и 14 имеем оценку

$$
\left\|\beta_{k}(x)-l(h)(x)\right\|_{\infty, \Omega}^{2}=\left\|\beta_{k}(x)-1\right\|_{\infty, \Omega}^{2} \leqslant \frac{\text { const }}{\left(\operatorname{Re} \lambda_{k}\right)^{2}}=\frac{\text { const }}{\lambda_{k}^{2}} .
$$

Используя асимптотику (8.15) для собственных значений задачи (8.5), выводим

$$
\left\|\psi_{k}-e_{k}\right\|_{2, \Omega}^{2}=\left\|\left(\beta_{k}-1\right) e_{k}\right\|_{2, \Omega}^{2} \leqslant\left\|\beta_{k}(x)-1\right\|_{\infty, \Omega}^{2} \leqslant \frac{\text { const }}{\lambda_{k}^{2}} \sim \frac{\text { const }}{k^{4 / n}} .
$$

Отсюда получаем, что для размерностей $n=1,2,3$ сходится числовой ряд

$$
\sum_{k=1}^{\infty}\left\|\psi_{k}-e_{k}\right\|_{2, \Omega}^{2} \leqslant \sum_{k=1}^{\infty}\left\|\beta_{k}-1\right\|_{\infty, \Omega}^{2}<\infty
$$

т.е. система $\left\{\psi_{k}(x)\right\}$ квадратично близка к ортонормированному базису $\left\{e_{k}(x)\right\}$. Выполнены все условия теоремы 2, а тогда по следствию $9\left\{\psi_{k}(x)\right\}$ является базисом Рисса в $L_{2}(\Omega)$. Следовательно, это базис Бари (по терминологии М. Г. Крейна, см. [38; гл. 6, §3]). Следствие 10 доказано.

Следствие 10 подчеркивает ключевую роль асимптотического соотношения (8.15) для наличия у системы $\left\{\psi_{k}(x)\right\}$ свойства базисности Бари. Примеры показывают, что для $n=4$ при выполнении всех остальных условий следствия 10 система $\left\{\psi_{k}(x)\right\}$ в общем случае не является базисом Бари.

ПримеР 2. Для определенности рассмотрим случай финального наблюдения в точке $t=T$. В этом случае оператор переопределения в (8.5) имеет вид $l(u)=\int_{0}^{T} u(x, t) d \mu(t) \equiv u(x, T)$. Выберем для удобства $T=1$. При этом функция $\mu(t)$ определяется условиями $\mu(t)=0$ для всех $t<1$ и $\mu(t)=1$ для всех $t \geqslant 1$. Тогда система функций (8.6) примет вид

$$
\psi_{k}(x)=\lambda_{k} \int_{0}^{1} e^{-\lambda_{k}(1-\tau)} h(x, \tau) d \tau e_{k}(x) \equiv \beta_{k}(x) e_{k}(x) \quad \text { при всех } k=1,2, \ldots
$$

Здесь $\left\{e_{k}(x)\right\}$ - это ортонормированный базис в $L_{2}(\Omega)$ из собственных функций задачи (8.5), а $\left\{\lambda_{k}\right\}$ - соответствующие собственные значения этой задачи, взятые с учетом кратности. В качестве $h(x, t)$ возьмем скалярную функцию одной переменной $h(t)=t, t \in[0,1]$, и найдем числа

$$
\beta_{k}:=\lambda_{k} \int_{0}^{1} e^{-\lambda_{k}(1-\tau)} \tau d \tau=1-\frac{1}{\lambda_{k}}+\frac{1}{\lambda_{k}} e^{-\lambda_{k}}
$$


Напомним, что в условиях этого параграфа $\lambda_{k}>0, \lambda_{k} \rightarrow+\infty, k \rightarrow \infty$. Ясно, что $\beta_{k}>0$ при всех $k \in \mathbb{N}$, а по лемме $13 \beta_{k} \rightarrow l(h)=h(1)=1$. Поэтому найдутся числа $b_{0}, b_{1}$ такие, что $0<b_{0}=\inf \beta_{n} \leqslant \beta_{k} \leqslant \sup \beta_{n}=b_{1}, k \in \mathbb{N}$. Рассмотрим систему $\psi_{k}(x)=\beta_{k} e_{k}(x)$ для $k=1,2, \ldots$ и докажем, что $\left\{\psi_{k}(x)\right\}-$ базис Рисса в $H=L_{2}(\Omega)$.

Введем оператор $U: H \rightarrow H$ по правилу $U f:=\sum_{k=1}^{\infty} \beta_{k} f_{k} e_{k}$ для любого $f \in H, f=\sum_{k=1}^{\infty} f_{k} e_{k}$, где $f_{k}=\left(f, e_{k}\right)$. Так как при всех $k \in \mathbb{N}$ справедливы неравенства $0 \leqslant \beta_{k}\left|f_{k}\right| \leqslant b_{1}\left|f_{k}\right|$, а $\|f\|^{2}=\sum_{k=1}^{\infty}\left|f_{k}\right|^{2}<\infty$, то сходится числовой ряд $\sum_{k=1}^{\infty}\left|\beta_{k}\right|^{2}\left|f_{k}\right|^{2}$. Следовательно, $U f \in H$, и определение корректно. Отсюда имеем $\|U f\|^{2}=\sum_{k=1}^{\infty}\left|\beta_{k}\right|^{2}\left|f_{k}\right|^{2} \leqslant\left(\sup \left|\beta_{k}\right|\right)^{2}\|f\|^{2}=\left(b_{1}\right)^{2}\|f\|^{2}$, т.е. оператор $U \in \mathscr{L}(H)$. Кроме того, из определения оператора $U$ очевидно, что выполнены равенства $U e_{k}=\psi_{k}, k \in \mathbb{N}$. Докажем, что его ядро $\operatorname{Ker} U$ содержит только нулевой элемент. Пусть элемент $f$ принадлежит $H$ и удовлетворяет уравнению $U f=\theta$, т.е. $\sum_{k=1}^{\infty} \beta_{k} f_{k} e_{k}=\theta$. Поскольку $\left\{e_{k}\right\}-$ базис в $H$, то $\beta_{k} f_{k}=0$, откуда (так как $\left.\beta_{k} \neq 0\right) f=\theta$. Найдем образ оператора $U$. Возьмем произвольный элемент $g \in H$ и разложим его по базису $\left\{e_{k}\right\}$ : $g=\sum_{k=1}^{\infty} g_{k} e_{k}$, где $g_{k}=\left(g, e_{k}\right)$. Для $g$ определим $f$ по следующей формуле: $f=\sum_{k=1}^{\infty}\left(g_{k} / \beta_{k}\right) e_{k}$. Элемент $f \in H$, поскольку сходится числовой ряд $\|f\|^{2}=\sum_{k=1}^{\infty}\left|g_{k}\right|^{2} /\left|\beta_{k}\right|^{2} \leqslant\left(\sup 1 /\left|\beta_{k}\right|\right)^{2}\|g\|^{2}=\left(1 / b_{0}\right)^{2}\|g\|^{2}$. Так как из построения $f$ ясно, что $U f=g$, то образ оператора совпадает со всем $H$. Отсюда по теореме Банаха об обратном операторе (см. [22; гл. 2, §2, теорема 2]) $U^{-1} \in \mathscr{L}(H)$. Следовательно, $\left\{\psi_{k}\right\}$ - базис Рисса. Отметим, что для области $\Omega$ с гладкой границей $\partial \Omega\left(\partial \Omega \in C^{2}\right)$ базисность Рисса этой системы вытекает из следствия 9. Чтобы не ограничивать область действия примера, мы доказали это независимо.

Докажем, что система $\left\{\psi_{k}\right\}$ не является базисом Бари, т.е. не является квадратично близкой ни к какому ортонормированному базису в $H$. По теореме 3.3 работы [38; гл. 6] для этого достаточно установить, что расходится следующий ряд $\sum_{j, k=1}^{\infty}\left|\left(\psi_{j}, \psi_{k}\right)-\delta_{j k}\right|^{2}$. Так как $\beta_{k}-$ это числа, то скалярные произведения $\left(\psi_{j}, \psi_{k}\right)$ легко вычисляются: $\left(\psi_{j}, \psi_{k}\right)=\beta_{j} \beta_{k} \delta_{j k}$. Поэтому ряд преобразуется к виду

$$
\sum_{j, k=1}^{\infty}\left|\left(\psi_{j}, \psi_{k}\right)-\delta_{j k}\right|^{2}=\sum_{k=1}^{\infty}\left|\beta_{k}^{2}-1\right|^{2}
$$

Из представления для $\beta_{k}($ см. (8.16)) имеем

$$
\left|\beta_{k}^{2}-1\right|^{2}=\left|\beta_{k}-1\right|^{2} \cdot\left|\beta_{k}+1\right|^{2} \sim \frac{4}{\left|\lambda_{k}\right|^{2}} \sim \frac{\text { const }}{k^{4 / n}} \quad \text { при } k \rightarrow \infty,
$$

где использована асимптотика (8.15). Поэтому при $n=4$ исследуемый числовой ряд (8.17) расходится, и $\left\{\psi_{k}\right\}$ не является базисом Бари в $H$.

\section{5. Заключительные замечания.}

ЗАмЕчАниЕ 13. Если обратная задача (8.1)-(8.3) корректна, то по теореме 10 система $\left\{\psi_{k}\right\}$ является базисом Рисса в $L_{2}(\Omega)$. Теорема 9 при этом дает возможность найти биортогональную систему $\left\{g_{k}(x)\right\}$ как решение некоторой 
последовательности обратных задач. Наличие системы $\left\{g_{k}(x)\right\}$ позволяет записать для решения обратной задачи следующую формулу:

$$
f=\sum_{k=1}^{\infty}\left(f, \psi_{k}\right) g_{k}
$$

Коэффициенты $\alpha_{k}=\left(f, \psi_{k}\right)$ этого ряда в силу равенств $(8.9)$ имеют простой вид $\alpha_{k}=\left(-L_{0} \chi, e_{k}\right)=\lambda_{k}\left(\chi, e_{k}\right)$, т.е. вычисляются по заданной функции $\chi(x)$ из условия наблюдения (8.3), при этом ряд (8.18) сходится в $L_{2}(\Omega)$. Функция $u=u(x, t ; f)$ находится как решение прямой задачи $(8.1),(8.2)$ с этим $f \in L_{2}(\Omega)$.

ЗАмЕчАНИЕ 14. Отметим один частный случай, когда вопрос о полноте и базисности системы $\left\{\psi_{k}\right\}$, а следовательно, о единственности и корректности обратной задачи, может быть решен полностью. Предположим, что $h=h(t)$ не зависит от пространственной переменной и $h \in W_{2}^{1}(0, T)$. Тогда

$$
\beta_{k}=\lambda_{k} \int_{0}^{T}\left(\int_{0}^{t} e^{-\lambda_{k}(t-\tau)} h(\tau) d \tau\right) d \mu(t)
$$

- это числовая последовательность и, очевидно, $\left\{\psi_{k}(x)\right\}=\left\{\beta_{k} e_{k}(x)\right\}$ полна только тогда, когда все $\beta_{k} \neq 0$. Биортогональная система $\left\{g_{k}\right\}$ в этом случае выписывается явно $\left\{g_{k}\right\}=\left\{\left(1 / \beta_{k}\right) e_{k}\right\}$. По лемме $13 \beta_{k} \rightarrow l(h)$ при $k \rightarrow \infty$. Поэтому, если $l(h) \neq 0$ и все $\beta_{k} \neq 0$, то $\left\{\psi_{k}\right\}$ - базис Рисса в $L_{2}(\Omega)$ (см. пример 2). Таким образом, из полноты системы $\left\{\psi_{k}\right\}$ в случае, когда $\beta_{k}$ - это скаляры, легко следует базисность Рисса, и тогда по теореме 10 получаем корректность обратной задачи. Этот факт есть проявление свойства фредгольмовости, доказанного в теореме 1.

Если же среди чисел $\beta_{k}$ имеются нули, то, очевидно, система $\left\{\psi_{k}\right\}$ не обладает свойством полноты. Единственности решения обратной задачи тоже нет. Действительно, пусть, например, $\beta_{1}=0$. Тогда пара функций $f(x)=e_{1}(x)$ и $u(x, t)=\int_{0}^{t} e^{-\lambda_{1}(t-\tau)} h(\tau) d \tau e_{1}(x)$ является (нетривиальным) решением однородной обратной задачи $(8.1)-(8.3)$ с $\chi(x)=0$ в $\Omega$. При проверке последнего условия необходимо учесть, что

$$
\beta_{1}:=\lambda_{1} \int_{0}^{T}\left(\int_{0}^{t} e^{-\lambda_{1}(t-\tau)} h(\tau) d \tau\right) d \mu(t)=0 .
$$

По теоремам 7 и 8 вопрос о существовании (единственности) решения обратной задачи эквивалентно сводится соответственно к разрешимости (единственности решения) проблемы моментов (8.11). Для скалярного случая она запишется в виде системы с диагональной матрицей относительно неизвестных величин $\left(f, e_{k}\right)$

$$
\left(f, \psi_{k}\right) \equiv \beta_{k}\left(f, e_{k}\right)=\alpha_{k} \quad \text { при } \quad k=1,2, \ldots
$$

Из вида этой системы непосредственно следуют такие утверждения.

1. Решение (8.19) единственно только тогда, когда все $\beta_{k} \neq 0$.

2. Если для некоторых $k \in \mathbb{N} \quad \beta_{k}=0$, то для разрешимости (8.19) необходимо выполнение равенств $\alpha_{k}=0$ при тех же значениях $k$. Отметим, что множество таких натуральных $k$ может быть и пустым. 
3. Если для некоторых $k \in \mathbb{N} \quad \beta_{k}=0$ и необходимое условие разрешимости (см. 2) выполнено, то решение (8.19) существует только тогда, когда сходится числовой ряд $\sum_{k}\left|\alpha_{k} / \beta_{k}\right|^{2}$, где суммирование проводится по всем $k \in \mathbb{N}$, для которых $\beta_{k} \neq 0$. Это решение будет заведомо не единственным. Оно находится с точностью до элемента $f_{0}$, принадлежащего замыканию линейной оболочки тех $e_{k}$, для которых $\beta_{k}=0$.

Автор благодарит А. И. Прилепко за внимание к настоящей работе, а участников семинара по обратным задачам за полезное обсуждение ее результатов.

\section{Список литературы}

[1] А. И. Прилепко, "Обратные задачи теории потенциала (эллиптические, параболические, гиперболические уравнения и уравнения переноса)", Матем. заметки, 14:5 (1973), 755-767; англ. пер.: A. I. Prilepko, "Reports delivered at a general meeting of the mathematics division, Academy of Sciences of the USSR", Math. Notes, 14:5 (1973), 990-996.

[2] В. М. Исаков, "Об одном классе обратных задач для параболических уравнений”, Докл. АН СССР, 263:6 (1982), 1296-1299; англ. пер.: V. M. Isakov, "On a class of inverse problems for parabolic equations", Soviet Math. Dokl., 25:2 (1982), 519-521.

[3] А. И. Прилепко, В. В. Соловьев, "Теоремы разрешимости и метод Ротэ в обратных задачах для уравнений параболического типа. II”, Дифферени. уравнения, 23:11 (1987), 1971-1980; англ. пер.: A. I. Prilepko, V. V. Solov'ev, "Solvability theorems and Rothe's method for inverse problems for a parabolic equation. II", Differential Equations, 23:11 (1987), 1341-1349.

[4] В. В. Соловьев, "О разрешимости обратной задачи определения источника с переопределением на верхней крышке для параболического уравнения", Дифферени. уравнения, 25:9 (1989), 1577-1583; англ. пер.: V.V. Solov'ev, "Solvability of the inverse problem of finding a source, using overdetermination on the upper base for a parabolic equation", Differential Equations, 25:9 (1989), 1114-1119.

[5] Ю. С. Эйдельман, "Единственность решения обратной задачи для дифференциального уравнения в банаховом пространстве", Дифферени. уравнения, 23:9 (1987), 1647-1649.

[6] Д. Г. Орловский, "K задаче определения параметра эволюционного уравнения", Дифферени. уравнения, 26:9 (1990), 1614-1621; англ. пер.: D. G. Orlovskiı̌, "Determination of a parameter of an evolution equation", Differential Equations, 26:9 (1990), 1201-1207.

[7] V. Isakov, "Inverse parabolic problems with the final overdetermination", Comm. Pure Appl. Math., 44:2 (1991), 185-209.

[8] А. И. Прилепко, А.Б. Костин, "О некоторых обратных задачах для параболических уравнений с финальным и интегральным наблюдением", Матем. сб., 183:4 (1992), 49-68; англ. пер.: А. I. Prilepko, А. B. Kostin, "On certain inverse problems for parabolic equations with final and integral observation", Russian Acad. Sci. Sb. Math., 75:2 (1993), 473-490.

[9] А. И. Прилепко, А. Б. Костин, "Оценка спектрального радиуса одного оператора и разрешимость обратных задач для эволюционных уравнений", Матем. заметки, 53:1 (1993), 89-94; англ. пер.: А. I. Prilepko, A. B. Kostin, "Estimation of the spectral radius of an operator and the solvability of inverse problems for evolution equations", Math. Notes, 53:1 (1993), 63-66.

[10] А. И. Прилепко, И. В. Тихонов, "Восстановление неоднородного слагаемого в абстрактном эволюционном уравнении", Изв. РАН. Сер. матем., 58:2 (1994), 
167-188; англ. пер.: A. I. Prilepko, I. V. Tikhonov, "Recovery of the nonhomogeneous term in an abstract evolution equation", Russian Acad. Sci. Izv. Math., 44:2 (1995), 373-394.

[11] И.В. Тихонов, Обратные задачи для эволючионных уравнений и приложения $\kappa$ уравнению переноса, Дис. ... канд. физ.-матем. наук, 1993.

[12] А. И. Прилепко, И. В. Тихонов, "Принцип позитивности решения в линейной обратной задаче и его применение к коэффициентной задаче теплопроводности", Докл. РАН, 364:1 (1999), 21-23; англ. пер.: А. I. Prilepko, I. V. Tikhonov, "The principle of the positiveness of a solution to a linear inverse problem and its application to the coefficient heat conduction problem", Dokl. Math., 59:1 (1999), 14-16.

[13] A. I. Prilepko, D. G. Orlovsky, I. A. Vasin, Methods for solving inverse problems in mathematical physics, Monogr. Textbooks Pure Appl. Math., 231, Marcel Dekker, New York, 2000.

[14] А. И. Прилепко, Д. С. Ткаченко, "Фредгольмовость и корректная разрешимость обратной задачи об источнике с интегральным переопределением", Ж. въчисл. матем. и матем. физ., 43:9 (2003), 1392-1401; англ. пер.: А. I. Prilepko, D. S. Tkachenko, "The Fredholm property and the well-posedness of the inverse source problem with integral overdetermination", Comput. Math. Math. Phys., 43:9 (2003), $1338-1347$.

[15] В.Л.Камынин, “Об обратной задаче определения правой части в параболическом уравнении с условием интегрального переопределения", Матем. заметки, 77:4 (2005), 522-534; англ. пер.: V. L. Kamynin, "On the inverse problem of determining the right-hand side of a parabolic equation under an integral overdetermination condition", Math. Notes, 77:4 (2005), 482-493.

[16] А.И. Прилепко, "Метод полугрупп решения обратных, нелокальных и неклассических задач. Прогноз-управление и прогноз-наблюдение эволюционных уравнений. I", Дифферени. уравнения, 41:11 (2005), 1560-1571; англ. пер.: A.I. Prilepko, "The semigroup method for inverse, nonlocal, and nonclassical problems. Prediction-control and prediction-observation for evolution equations. I", Differ. Equ., 41:11 (2005), 1635-1646.

[17] А. И. Прилепко, А. Б. Костин, "Об обратных задачах определения коэффициента в параболическом уравнении. I", Сиб. матем. журн., 33:3 (1992), 146-155; англ. пер.: А. I. Prilepko, А. B. Kostin, "Inverse problems of the determination of the coefficient in parabolic equations. I", Siberian Math. J., 33:3 (1992), 489-496.

[18] А.И. Прилепко, И.А. Васин, "Некоторые обратные начально-краевые задачи для нестационарных линеаризованных уравнений Навье-Стокса", Дифферени. уравнения, 25:1 (1989), 107-117; англ. пер.: А. I. Prilepko, A. B. Kostin, "Inverse initial-boundary-value problems for linearized nonstationary Navier-Stokes equations", Differential Equations, 25:1 (1989), 85-92.

[19] A. I. Kozhanov, R. R. Safiullova, "Linear inverse problems for parabolic and hyperbolic equations", J. Inverse Ill-Posed Probl., 18:1 (2010), 1-24.

[20] А. Б. Костин, "Базисность одной системы функций, связанной с обратной задачей нахождения источника", Дифферени. уравнения, 44:2 (2008), 246-256; англ. пер.: A. B. Kostin, "Basis property of a system of functions related to the inverse problem of finding the source", Differential Equations, 44:2 (2008), 256-266.

[21] О.А. Ладыженская, В.А. Солонников, Н.Н. Уральцева, Линейные и квазилинейные уравнения параболического типа, Наука, М., 1967; англ. пер.: O. A. Ladyzhenskaya, V. A. Solonnikov, N. N. Ural'tseva, Linear and quasi-linear equations of parabolic type, Transl. Math. Monogr., 23, Amer. Math. Soc., Providence, RI, 1968.

[22] Н. Данфорд, Дж. Шварц, Линейнье операторы. Часть 1. Общал теория, ИЛ, M., 1962; пер. с англ.: N. Dunford, J. T. Schwartz, Linear operators. I. General theory., Pure Appl. Math., 7, Intersci. Publ., New York-London, 1958. 
[23] И.П. Натансон, Теория функиий вещественной переменной, Лань, СПб., 1999; англ. пер. 1-го изд.: I. P. Natanson, Theory of functions of a real variable, Frederick Ungar Publ. Co., New York, 1955.

[24] G. M. Lieberman, Second order parabolic differential equations, World Scientific, Singapore, 2005.

[25] О.А. Ладыженская, Н.Н. Уральцева, Линейные и квазилинейные уравнения эллиптического типа, 2-е изд., Наука, М., 1973; англ. пер. 1-го изд.: O.A. Ladyzhenskaya, N.N. Ural'tseva, Linear and quasilinear elliptic equations, Math. Sci. Engrg., 46, Academic Press, New York-London, 1968.

[26] В. П. Михайлов, Дифференииальные уравнения в частных производных, 2-е изд., Наука, М., 1983; англ. пер. 1-го изд.: V. Р. Mikhajlov, Partial differential equations, Mir, Moscow, 1978.

[27] N. S. Trudinger, "Pointwise estimates and quasilinear parabolic equations", Comm. Pure Appl. Math., 21 (1968), 205-226.

[28] М. Г. Крейн, М. А. Рутман, “Линейные операторы, оставляющие инвариантным конус в пространстве Банаха", УМН, 3:1(23) (1948), 3-95.

[29] Функииональный анализ, 2-е изд., ред. С. Г. Крейн, Наука, М., 1972; англ. пер. 1-го изд.: G.F. Votruba, L.F. Boron (eds.), Functional analysis, Wolters-Noordhoff Publ., Groningen, 1972.

[30] M. Chicco, "Principio di massimo per soluzioni di problemi al contorno misti per equazioni ellittiche di tipo variazionale", Boll. Un. Mat. Ital. (4), 3 (1970), 384-394.

[31] Д. Гилбарг, Н.С. Трудингер, Эллиптические дифференииальные уравнения с частными производными второго порядка, Наука, М., 1989; пер. с англ.: D. Gilbarg, N.S. Trudinger, Elliptic partial differential equations of second order, Grundlehren Math. Wiss., 224, Springer-Verlag, Berlin, 1983.

[32] А.Б. Костин, "Разрешимость одной проблемы моментов и ее связь с параболической обратной задачей", Вестн. Моск. ун-та. Сер. 15. Вычислит. матем. и киберн., 1995, № 1, 28-33.

[33] Д. Г. Орловский, "Определение параметра параболического уравнения в гильбертовой структуре", Матем. заметки, 55:3 (1994), 109-117; англ. пер.: D. G. Orlovskii, "Determination of a parameter of a parabolic equation in Hilbert's structure", Math. Notes, 55:3 (1994), 312-317.

[34] А.И. Прилепко, И.В. Тихонов, "Обратная задача с финальным переопределением для абстрактного эволюционного уравнения в упорядоченном банаховом пространстве", Функи. анализ и его прил., 27:1 (1993), 81-83; англ. пер.: A.I. Prilepko, I. V. Tikhonov, "An inverse problem with final time additional condition for an abstract evolution equation in an ordered Banach space", Funct. Anal. Appl., 27:1 (1993), 68-70.

[35] А. И. Прилепко, А. Б. Костин, "О некоторых задачах восстановления граничного условия для параболического уравнения. I”, Дифферени. уравнения, 32:1 (1996), 107-116; англ. пер.: А. В. Kostin, A. I. Prilepko, "On some problems of restoration of a boundary condition for a parabolic equation. I", Differential Equations, 32:1 (1996), $113-122$.

[36] В.П. Михайлов, А. К. Гущин, “Дополнительные главы курса «Уравнения математической физики»", Лекц. курсы НОЦ, 7, МИАН, М., 2007, 3-144.

[37] А. М. Денисов, Введение в теорию обратных задач, МГУ, М., 1994; англ. пер.: A. M. Denisov, Elements of the theory of inverse problems, Inverse Ill-posed Probl. Ser., VSP, Utrecht, 1999.

[38] И.Ц. Гохберг, М.Г. Крейн, Введение в теорию линейных несамосопряженных операторов в гильбертовом пространстве, Наука, М., 1965; англ. пер.: I. C. Gohberg, M. G. Kreln, Introduction to the theory of linear nonselfadjoint operators, Transl. Math. Monogr., 18, Amer. Math. Soc., Providence, RI, 1969. 
[39] T. Carleman, "Über die asymptotische Verteilung der Eigenwerte partieller Differentialgleichungen", Ber. Verh. Sachs. Akad. Leipzig, 88 (1936), 119-132.

[40] М.Ш. Бирман, М. З. Соломяк, "Асимптотика спектра дифференциальных уравнений”, Итоги науки и техн. Сер. Мат. анал., 14, ВИНИТИ, М., 1977, 5-58; англ. пер.: M. Sh. Birman, M.Z. Solomyak, "Asymptotic behavior of the spectrum of differential equations", J. Soviet Math., 12:3 (1979), 247-283.

\author{
Андрей Борисович Костин \\ (Andrey B. Kostin) \\ Национальный исследовательский ядерный \\ университет "МИФИ", г. Москва \\ E-mail: abkostin@yandex.ru
}

Поступила в редакцию 16.01.2012 и 17.06.2013 